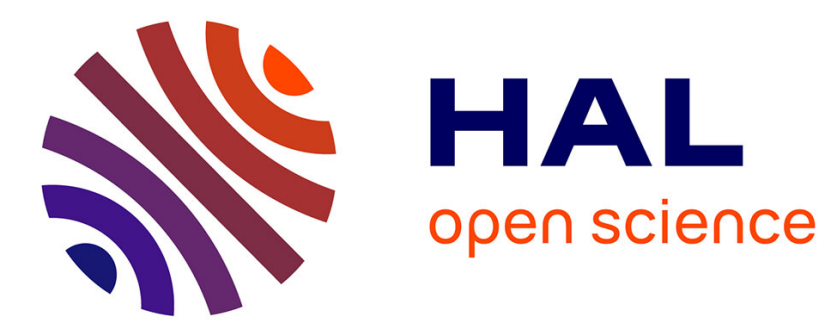

\title{
Continuum theory of evolving dislocation fields
}

\author{
Radan Sedlacek, Cornelia Schwarz, Jan Kratochvil, Ewald Werner
}

\section{To cite this version:}

Radan Sedlacek, Cornelia Schwarz, Jan Kratochvil, Ewald Werner. Continuum theory of evolving dislocation fields. Philosophical Magazine, 2007, 87 (08-09), pp.1225-1260. 10.1080/14786430600972921 . hal-00513770

\section{HAL Id: hal-00513770 \\ https://hal.science/hal-00513770}

Submitted on 1 Sep 2010

HAL is a multi-disciplinary open access archive for the deposit and dissemination of scientific research documents, whether they are published or not. The documents may come from teaching and research institutions in France or abroad, or from public or private research centers.
L'archive ouverte pluridisciplinaire HAL, est destinée au dépôt et à la diffusion de documents scientifiques de niveau recherche, publiés ou non, émanant des établissements d'enseignement et de recherche français ou étrangers, des laboratoires publics ou privés. 


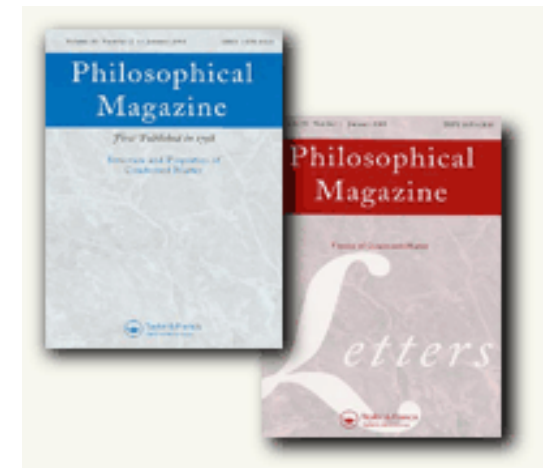

\section{Continuum theory of evolving dislocation fields}

\begin{tabular}{|r|l|}
\hline Journal: & Philosophical Magazine \& Philosophical Magazine Letters \\
\hline Manuscript ID: & TPHM-06-Feb-0039.R1 \\
\hline Journal Selection: & Philosophical Magazine \\
\hline Date Submitted by the & 19-Jul-2006 \\
\hline Complete List of Authors: & $\begin{array}{l}\text { Sedlacek, Radan; Technische Universitaet Muenchen } \\
\text { Schwarz, Cornelia; Technische Universitaet Muenchen } \\
\text { Kratochvil, Jan; Czech Technical University, Faculty of Civil } \\
\text { Engineering, Dept of Physics } \\
\text { Werner, Ewald; Technische Universitaet Muenchen }\end{array}$ \\
\hline Keywords: & dislocations, plasticity \\
\hline Keywords (user supplied): & line-tension, size-effect \\
\hline $\begin{array}{l}\text { Note: The following files were submitted by the author for peer review, but cannot be converted } \\
\text { to PDF. You must view these files (e.g. movies) online. }\end{array}$ \\
\hline Sedlacek 230806.tex \\
\hline
\end{tabular}

\section{S ScholaroNE \\ Manuscript Central}




\title{
Continuum theory of evolving dislocation fields
}

\author{
RADAN SEDláČEK*†, Cornelia SchWarz $\dagger$, \\ JAN KratochVÍl $\ddagger$ AND EWALd WeRner $\dagger$ \\ $\dagger$ Technische Universität München, Fakultät für Maschinenwesen \\ Lehrstuhl für Werkstoffkunde und Werkstoffmechanik \\ Boltzmannstr. 15, 85747 Garching, Germany \\ $\ddagger$ Czech Technical University, Faculty of Civil Engineering, \\ Department of Physics, \\ Thákurova 7, 16629 Prague, Czech Republic
}

\begin{abstract}
Continuum theory of moving dislocations is used to set up a non-local constitutive law for crystal plasticity in the form of partial differential equations for evolving dislocation fields. The concept of single-valued dislocation fields that enables to keep track of the curvature of the continuously distributed gliding dislocations with line tension is utilized. The theory is formulated in the Eulerian as well as in so-called dislocation-Lagrangian forms. The general theory is then specialized to a form appropriate to formulate and solve plane-strain problems of continuum mechanics. The key equation of the specialized theory is identified as a transport equation of diffusion-convection type. The numerical instabilities resulting from the dominating convection are eliminated by resorting to the dislocation-Lagrangian approach. Several examples illustrate the application of the theory.
\end{abstract}

Keywords: plasticity, dislocations, line-tension, size-effect

\section{Introduction}

Motivated mostly by the experimental finding that the response of plastically deforming materials on the length scale of some $10 \mu \mathrm{m}$ and below is size dependent $[1,2,3]$, the inter-

\footnotetext{
*Corresponding author. Email: sedlacek@lam.mw.tum.de
} 
est in non-local constitutive equations for plasticity has been revived over the past years. The efforts can be roughly divided into two groups. First, the classical phenomenological constitutive laws have been enriched by gradient terms that account for the length-scale dependence, see Hutchinson [4] and Needleman [5] for reviews. These laws can be fitted to experimental data and used efficiently for numerical computations, but they do not provide any deeper insight in the mechanisms leading to the observed length-scale dependence of plastic deformation. Accordingly, the predictive power of these laws is limited. Second, dislocation dynamics has emerged recently as a candidate for a physically-based non-local constitutive law of plastic deformation. Originally, the simulations of discrete dislocation dynamics were devoted to studies of the mechanisms of dislocation interactions and patterning $[6,7]$. Only recently, the coupling of discrete dislocation dynamics with continuum mechanics has been accomplished. The discrete dislocation dynamics was used firstly as a constitutive law in two-dimensional simulations of plastic deformation resulting from motion of straight parallel dislocations [8], afterwards also in a general framework using curved dislocations in three dimensions $[9,10]$. In these simulations, the size effects result naturally from dislocation interactions. However, there are still unsolved problems related to the coupling of the dislocation dynamics with continuum mechanics, and, moreover, these computations are very demanding. It has been noted that dislocation dynamics may never become efficient enough to make computationally practical the use of discrete dislocation simulations as material constitutive functions for continuum finite element simulations [11].

A way out of the dilemma could be a density-based modelling of dislocations used as a non-local constitutive law for plasticity. In such an approach, the advantages of the phenomenological laws (computational efficiency) and of dislocation dynamics (physical transparency and predictive power) should merge. The starting point to such an approach can be the statistical averaging of the discrete dislocation dynamics foreseen long ago by Kröner [12]. The statistical averaging was successfully accomplished by Groma $[13,14]$ in the special case of straight parallel dislocations. The resulting continuum theory has been used as constitutive law in two-dimensional simulations of plastic deformation [15]. Unfortunately, a corresponding rigorous theory for curved dislocations is still lacking. An alternative approach to the density-based modelling of dislocations is to start with the continuum theory of dislocations that was developed originally to deal with the description of incompatibility and internal stresses resulting from a prescribed dislocation distribution [16]. In the present work, we further develop the non-local continuum theory of evolving dislocation fields based on the continuum theory of moving dislocations $[16,17,18,19,20,21]$ that we have proposed earlier $[22,23,24,25]$. The length-scale dependency of this approach results from the line tension of the curved dislocations squeezing through small deforming volumes. The theory serves as a non-local dislocation-based constitutive law for plastic deformation that is coupled to continuum mechanics as shown in Fig. 1. [Insert Fig. 1] Although the constitutive law and the continuum mechanics framework present an indivisible unit, in this paper we focus our attention on the dislocation part. Attention is paid to the development of the theory as well as 
to problems related to its numerical implementation. We show that the implementation of the theory results in a diffusion-convection transport equation with dominant convection. Such equations are known to cause spurious oscillations and instabilities when solved by standard numerical methods [26]. Special treatment is required, for instance the streamline-upwind/Petrov-Galerkin or least-squares/Galerkin, see [26] for a review, or mesh-free/particle-tracking as well as semi-Lagrangian/particle-transport methods, see e.g. [27]. Similar problems have been encountered when implementing other theories dealing with dislocation plasticity. For instance, the streamline upwinding was used by Arsenlis et al. [28] when implementing their edge-screw model, whereas the least-square Galerkin was utilized by Roy and Acharya [29] who dealt with equations quite similar to ours, however, resolving individual dislocations. The weighted essentially non-oscillatory method was used in the phase field modelling of individual dislocations by Xiang et al. [30]. Here we have developed to the so-called dislocation-Lagrangian approach, which is a kind of a particle-tracking method. This approach reflects the nature of the dislocation kinematics and is suitable to coupling with the continuum mechanics part of the problem in the sense indicated in Fig. 1.

The plan of the paper is as follows. The problems arising when one uses the continuum theory of dislocations to set up a non-local constitutive law for plastic deformation are analyzed in detail in Sec. 2. The general theory of evolving dislocation fields based on the concept of single-valued dislocation fields is reviewed in Sec. 3. Evolution equations for the individual variables (dislocation density and orientation) are set up in Sec. 4. In this Section, an application example is considered, showing that the evolution equations correctly account for the increasing length of expanding dislocation loops. The theory is presented in the Eulerian as well as in the so-called dislocation-Lagrangian form. In Section 5, the theory is specialized to one spatial dimension. The varying convective character of the resulting equations is analyzed in terms of the Péclet number. Another application example is presented, showing that the dislocation-Lagrangian formulation eliminates the spurious oscillations and instabilities in the numerical implementation of the present theory.

\section{Motivation}

There are three major problems to be overcome when developing non-local constitutive equations for plasticity based on the continuum theory of moving dislocations: (i) The continuum description itself eliminates the short-range elastic fields of the individual crystal dislocations, thus eliminating the short-range dislocation interactions responsible for the size effects. This problem is discussed in Sec.2.1. (ii) Because of the problem of averaging [31], the classical continuum theory of dislocations in its original form 
$[16,17,18,19,20,21]$ does not enable us to derive the plastic strain rate from the density and velocity of the continuously distributed dislocations. (iii) The dislocation curvature that is decisive for the self force, i.e. the short-range interaction of a curved dislocation with itself, cannot be determined in the framework of the classical theory. The interrelated problems (ii) and (iii) are discussed in Sec. 2.2.

\subsection{Elastic fields of continuously distributed dislocations}

Given a distribution of dislocations in a crystal in time $t$, the dislocation density tensor $\boldsymbol{\alpha}(\boldsymbol{x}, t)$ of the classical theory can be introduced as a sum of the tensor products taken over all the dislocation segments present in a volume $\mathrm{d} V$ which is positioned at $\boldsymbol{x}$,

$$
\boldsymbol{\alpha}=\frac{1}{\mathrm{~d} V} \sum_{i} \boldsymbol{b}^{(i)} \otimes \boldsymbol{\xi}^{(i)} \mathrm{d} s^{(i)},
$$

where $\boldsymbol{b}^{(i)}$ is the Burgers vector, $\boldsymbol{\xi}^{(i)}$ is a unit tangent to the dislocation line, and $\mathrm{d} s^{(i)}$ is the length of the $i$-th dislocation segment. Applied on a test area $\mathrm{d} \boldsymbol{a}$, the dislocation density tensor yields the resulting Burgers vector $\mathrm{d} \boldsymbol{B}$ of all the dislocations piercing through this area,

$$
\mathrm{d} \boldsymbol{B}=\boldsymbol{\alpha} \mathrm{d} \boldsymbol{a} .
$$

This follows from the definition of a tensor as a linear transformation [32], namely, the tensor $\boldsymbol{b} \otimes \boldsymbol{\xi}$ transforms any vector in a vector parallel to $\boldsymbol{b}$,

$$
(\boldsymbol{b} \otimes \boldsymbol{\xi}) \mathrm{d} \boldsymbol{a}=\boldsymbol{b}(\boldsymbol{\xi} \cdot \mathrm{d} \boldsymbol{a}),
$$

where the scalar $\boldsymbol{\xi} \cdot \mathrm{d} \boldsymbol{a}$ is the projection of the dislocation line direction in the direction of a normal vector to the oriented surface element $\mathrm{d} \boldsymbol{a}$. The total length of the dislocation segments per unit volume, regardless of their Burgers vector and orientation, is represented by the scalar dislocation density $\varrho(\boldsymbol{x}, t)$ introduced as

$$
\varrho=\frac{1}{\mathrm{~d} V} \sum_{i} \mathrm{~d} s^{(i)} .
$$

Now recall the standard definition of Burgers vector of a dislocation [33],

$$
\boldsymbol{b}=\oint_{C} \boldsymbol{\beta}^{\mathrm{e}} \mathrm{d} \boldsymbol{l}
$$

where $\boldsymbol{\beta}^{\mathrm{e}}(\boldsymbol{x}, t)$ is the elastic distortion caused by the dislocation and the integration is performed along a closed curve $C$ encircling the dislocation line. The formal transition to a continuum description is accomplished by considering the limit case,

$$
\left.\begin{array}{rl}
\varrho(\boldsymbol{x}, t) & \rightarrow \infty \\
b & \rightarrow 0
\end{array}\right\} \quad \text { so that } \quad b \varrho(\boldsymbol{x}, t)=\operatorname{const}(\boldsymbol{x}, t)
$$


where $b$ is the Burgers vector magnitude. By this transition, the discrete dislocation lines have been smeared to a continuum. Since $b \rightarrow 0$, eq. (5), the elastic fields of the original individual dislocations, cf. eq. (4), are not accounted for in the continuum description ${ }^{1}$.

The dislocation density tensor can be introduced alternatively as the measure of incompatibility of the elastic and plastic deformation [16]. Consider a material distortion $\boldsymbol{\beta}(\boldsymbol{x}, t)$ introduced as

$$
\boldsymbol{\beta}=\operatorname{grad} \boldsymbol{u} .
$$

Being derived from a continuous and differentiable material displacement field $\boldsymbol{u}(\boldsymbol{x}, t)$, the material distortion is compatible,

$$
\operatorname{curl} \boldsymbol{\beta}=\mathbf{0} .
$$

In the small-strain approximation, the material distortion can be additively decomposed into a plastic and an elastic part,

$$
\boldsymbol{\beta}=\boldsymbol{\beta}^{\mathrm{p}}+\boldsymbol{\beta}^{\mathrm{e}}
$$

each of them being in general incompatible. A tensor $\boldsymbol{\alpha}(\boldsymbol{x}, t)$ can be introduced as the measure of incompatibility of the plastic distortion,

$$
\boldsymbol{\alpha}=-\operatorname{curl} \boldsymbol{\beta}^{\mathrm{p}} .
$$

We show that the tensor $\boldsymbol{\alpha}(\boldsymbol{x}, t)$ defined by relation (9) has the physical meaning described by eq. (2), i.e. the same one as the dislocation density tensor introduced in eq. (1). To this end, we express the total Burgers vector of all the continuously distributed dislocations piercing through an area $A$ which is bounded by a closed curve $C$. In analogy with eq. (4) we get

$$
\boldsymbol{B}=\oint_{C} \boldsymbol{\beta}^{\mathrm{e}} \mathrm{d} \boldsymbol{l}=-\int_{A} \operatorname{curl} \boldsymbol{\beta}^{\mathrm{p}} \mathrm{d} \boldsymbol{a}=\int_{A} \boldsymbol{\alpha} \mathrm{d} \boldsymbol{a} .
$$

Here we have used Stokes' theorem as well as eqs. (8) and (9). Since $A$ is arbitrary, eq. (10) yields the field equation (2). The resulting Burgers vector $\mathrm{d} \boldsymbol{B}(\boldsymbol{x}, t)$ of the continuously distributed dislocations can be non-zero since the product $b \varrho(\boldsymbol{x}, t)$ which implicitly enters the dislocation density tensor $\boldsymbol{\alpha}(\boldsymbol{x}, t)$ remains finite in the continuum description, eq. (5). Accordingly, as distinct from the short-range fields related to individual dislocations, the long-range elastic field of an assemble of dislocations is accounted for in the continuum description. It can be computed either directly from the dislocation density tensor in the framework of the theory of self-stresses [16], or indirectly, via coupling of the continuously distributed dislocations with continuum-mechanics framework.

The short-range elastic fields that are absent in the classical continuum description are decisive for the dislocation interactions that determine the size-dependent plastic response.

\footnotetext{
${ }^{1} \mathrm{~A}$ non-vanishing elastic distortion that fulfills the relation $\oint_{C} \boldsymbol{\beta}^{\mathrm{e}} \mathrm{d} \boldsymbol{l}=0$ is a compatible one, it can be derived from a potential (displacement) and as such it is not a field of a dislocation encircled by the curve $C$.
} 
One of the most important interactions, especially when plasticity of small confined volumes is considered, is the self force of curved dislocations. It can be reintroduced in the continuum description explicitly, by means of the line-tension concept, provided, the dislocation curvature can be determined. This approach, pioneered by Kratochvíl and Saxlová [34], and used e.g. by Sedláček et al. [35, 22], has been developed as a concise theory by Sedláček et al. [23, 24] and it is employed also here. As for the mutual dislocation interactions in a continuum description, a rigorous statistical approach using dislocation correlation functions has been developed for straight parallel dislocations by Groma et al. $[13,14]$. Although sketched in general terms long ago by Kröner [12], a rigorous statistical theory for general curved dislocations is still lacking [31]. In the present approach, we account for the mutual dislocation interactions only indirectly, using the standard Taylor formula for the yield stress,

$$
\hat{\tau}=\alpha \mu b \sqrt{\varrho},
$$

where $\alpha \in(0.1,0.6)$ is a phenomenological constant, $\mu$ is the elastic shear modulus, and $\varrho(\boldsymbol{x}, t)$ the scalar dislocation density, eq. (3). As distinct from the statistical approach of Groma et al. [14] that accounts for a length-scale effect caused by short-range interactions between dislocations, the Taylor relation (11) is length-scale independent. Other shortrange interactions can be considered in the continuum framework as well, depending on the specific problem at hand. For example, hardening by dipolar dislocation loops has been considered in the continuum framework by Sedláček and Kratochvíl [24].

\subsection{Incompleteness of the continuum description}

The greatest shortcoming of the continuum theory is that the dislocation density tensor $\boldsymbol{\alpha}(\boldsymbol{x}, t)$, eq. (1), eq. (9), measures the average dislocation density only [31]. For this reason, it is generally impossible to derive the plastic strain rate from the dislocation density described by the tensor $\boldsymbol{\alpha}(\boldsymbol{x}, t)$ and velocity $\boldsymbol{V}(\boldsymbol{x}, t)$ of the dislocations. Formally, a thirdorder dislocation velocity tensor can be introduced as $\boldsymbol{\alpha} \otimes \boldsymbol{V}$ [16]. However, as discussed e.g. by [20], if there is the same number of dislocations of Burgers vector $\boldsymbol{b}$ oriented along $\boldsymbol{\xi}$ and $-\boldsymbol{\xi}$ in the volume element used for the averaging, the resulting dislocation density tensor $\boldsymbol{\alpha}$ determined from eq. (1) vanishes, leading to a vanishing dislocation velocity tensor. However, the Peach-Koehler forces acting on the anti-parallel dislocations point in opposite directions in a given stress field, so that the dislocations glide in opposite directions, thus causing a non-vanishing plastic slip rate. The latter is not accounted for by the dislocation velocity tensor of the classical theory. This problem has been known for a long time and attempts have been made to circumvent it. Sorting dislocation segments according to their orientation, Kosevich $[18,19]$ has proposed a refined averaging of the dislocation density, whereas Mura [20, 21] speaks of sets or classes of dislocations of the same orientation. Considering distributions of dislocation segments in a phase space of position, orientation and velocity, El-Azab [36] set up a complete statistical mechanics theory based on a similar idea. 
However, there is still another problem related to the continuum description which is illustrated in Fig. 2. [Insert Fig. 2] Let us consider a plastically deforming channel bounded by elastic walls. Suppose, the plastic deformation in the channel has been caused by continuously distributed dislocation loops depicted in the left part of Fig. 2 . Describing these loops by the dislocation density tensor $\boldsymbol{\alpha}(\boldsymbol{x}, t)$, we are left with the so-called geometrically necessary dislocation content shown schematically in the right of Fig. 2. Because of the averaging inherent to the continuum approach, the positive and negative screw parts of the dislocation loops cancel each other. This is a great problem not only because the scalar density of the resulting geometrically necessary dislocations does not correspond to the original scalar density of the loops, but also because any information on the shape of the original loops has been lost. At the same time, it is the curvature of the loops that determines the self force which is decisive for the size-dependent plastic response in this case (Orowan bowing).

To resolve this problem, the refined averaging based on the local sorting of dislocation segments according to their orientation $[18,19,20,21,36]$ seems to be insufficient. The reason is that the information needed is a non-local one. Roughly speaking, we need to keep the information on the connectivity of the original dislocation lines in the continuum description. The dislocation curvature can then be determined from the orientation of the neighboring segments belonging to the same dislocation. To this end, the concept of single-valued dislocation fields has been proposed by Sedláček et al. [23, 24]. This concept, which is central to this paper as well, is recalled in the next Section.

\section{Single-valued dislocation fields}

In many cases of practical interest, especially in confined plasticity, but also in some self-organized dislocation structures, a real dislocation configuration can be approximated by a superposition of smooth dislocation fields where the dislocation orientation $\boldsymbol{\xi}(\boldsymbol{x}, t)$ of each field is a unique function of the position $\boldsymbol{x}$. In each volume element used for the averaging, the continuously distributed dislocations of these individual single-valued fields are parallel and have the same orientation. A typical example is shown in the left of Fig. 2, where the dislocation configuration can be considered as a local superposition of two single-valued fields. As shown by Sedláček et al. [23], such a dislocation configuration can be characterized by a distribution function $\phi(x, \vartheta)$, where $x$ is the coordinate across the channel and $\vartheta$ is the angle between this coordinate axis and the Burgers vector $\boldsymbol{b}$ of the loops, such that the integral over $\vartheta \in[0,2 \pi)$ yields the scalar density, $\int \phi(x, \vartheta) \mathrm{d} \vartheta=\varrho(x)$. The set $\operatorname{supp}(\phi)=\{(x, \vartheta): \phi(x, \vartheta) \neq 0\}$ forms a curve in the phase space $(x, \vartheta)$ of the position and orientation. As shown in [23], the dislocation configuration locally separates in the phase space into two single-valued fields, and we are able to follow the curved dislocation lines of 
each of the (locally) single-valued fields despite of the fact that there are two different orientations at each position $x$ across the channel, see Fig. 2 (left). The evolution of such a dislocation configuration described by the distribution function $\phi(x, \vartheta)$ has been computed in [23].

In applications, however, we prefer a more pragmatic approach. The dislocation configuration is separated into its single-valued components a priori and the individual fields are treated separately. For example, in the configuration shown in Fig. 2 (left), the 'upper' and 'lower' parts of the loops can be considered as the single-valued fields. In this way, many problems of practical interest have been solved, among others formation of a dipolar dislocation structure [24], shearing [25] and bending [37] of a thin film, as well as deformation of a composite material in plane strain, where the present method has been coupled with finite elements [38]. Because of its simplicity, this pragmatic approach is adopted in the present paper as well. Accordingly, we develop the theory directly for a single-valued field rather than for the distribution function considered in [23]. Before doing that, we note that the concept of single-valued dislocation fields is not well applicable to statistical dislocation distributions where the individual dislocations form fully disorganized structures - a problem that any deterministic approach based on the continuum theory of dislocations has to face.

The equations governing the evolution of single-valued dislocation fields have been inferred in a top-down manner from the standard continuum theory of dislocations $[16,17,18,19]$ by Sedláček et al. [23, 24]. Here, generalizing the approach of Mura [20, 21], we derive these equations from the bottom up which enables us a better insight in the physical meaning of the equations. Moreover, we prove some relations that these equations fulfil and that will be utilized in numerical implementations of the theory. We also present some illustrative application examples.

\subsection{Basic definitions}

Let us consider an evolving field of continuously distributed curved dislocations with Burgers vector $\boldsymbol{b}$ and unit tangent to the dislocation lines $\boldsymbol{\xi}(\boldsymbol{x}, t)$. The dislocations are distributed with scalar density (line length per volume) $\rho(\boldsymbol{x}, t)$. The field is assumed to be single-valued, that is, the orientation $\boldsymbol{\xi}(\boldsymbol{x}, t)$ is a unique function of its arguments. Strictly speaking, working within the continuum framework, we should not use the finite Burgers vector and density of the original dislocations, but only their product in the sense of eq. (5). Nevertheless, we will see that in all the field equations derived below, the Burgers vector and density appear in a product, so it is possible to use their original values. 
that is known as the glide plane of the dislocations. The vectors $\boldsymbol{s}$ and $\boldsymbol{m}$ define a glide system. In the small-strain approximation adopted, cf. eq. (8), the vectors $s$ and $\boldsymbol{m}$ are constant in space. The considerations here are restricted to planar dislocations confined to their glide planes. The dislocations glide with velocity $\boldsymbol{V}(\boldsymbol{x}, t)=$ $V \boldsymbol{\nu}$, where $V(\boldsymbol{x}, t)$ is the velocity magnitude and $\boldsymbol{\nu}(\boldsymbol{x}, t)$ is a unit vector in the glide plane, normal to the dislocation lines such that

$$
\xi \times \nu=m
$$

The scalar field of the local dislocation curvature $\kappa(\boldsymbol{x}, t)$ has been derived by Sedláček et al. [23] from the Frenet formula as

$$
\kappa=-\operatorname{div} \boldsymbol{\nu} .
$$

The glide velocity $\boldsymbol{V}(\boldsymbol{x}, t)$ can be determined from an equation of motion of a dislocation. Assuming an over-damped dislocation motion for simplicity, we consider a linear relationship between the forces acting on the dislocation and the velocity magnitude,

$$
B V=\left\{\begin{array}{rll}
\operatorname{sign} \tau(|\tau|-\hat{\tau}) b+T \kappa & \text { for } & |\tau|>\hat{\tau} \\
0 & \text { for } & |\tau| \leq \hat{\tau}
\end{array}\right.
$$

We note in passing that eq. (14) plays the role of a yield condition. Here, $B$ is a drag coefficient, $\tau(\boldsymbol{x}, t)$ is the resolved shear stress on the slip system,

$$
\tau=\boldsymbol{s} \cdot \boldsymbol{\sigma} \boldsymbol{m}
$$

$\boldsymbol{\sigma}(\boldsymbol{x}, t)$ being the stress tensor. The stress tensor comprises stresses due to mechanical boundary conditions as well as stresses due to the long-range dislocation interactions. Accounting indirectly for short-range interactions among dislocations, the yield stress $\hat{\tau}>0$ can be related to the dislocation density via Taylor relation $(11)^{2}$. The term $T \kappa$ accounts for the short-range interaction of a curved dislocation with itself. The line

\footnotetext{
${ }^{2} \mathrm{~A}$ more detailed relation distinguishing e.g. between the strength of coplanar and non-coplanar dislocation interactions could be set up as well.
} 
tension $T$ can be considered either orientation dependent [39], or constant, $T \approx \mu b^{2}$ [33]. The curvature $\kappa$ of the dislocation equals the curvature of the continuous field, eq. (13). Note that eq. (14) is an equation of motion of an individual dislocation that represents the behavior of the continuously distributed dislocations at the position $\boldsymbol{x}$ and in time $t$. This deviation from a strictly continuum description, eq. (5), is necessary, for only a dislocation with a non-vanishing Burgers vector can interact with the elastic field (the term $\tau b$ ) and possesses the line tension $T$. It is the straightening force $T \kappa$ in eq. (14) that introduces the length-scale dependency into the present theory.

We note in passing that other short-range dislocation interactions can be considered in eq. (14) as well. For instance, hardening by dipolar dislocation loops has been considered by Kratochvíl and Sedláček in a work on dislocation patterning [24]. Applying the approach of Groma et al. [14], Kratochvíl and Sedláček have considered the short-range interactions between parallel dislocation segments, which results in non-locality in the direction perpendicular to dislocation lines [40]. Kubin et al. [41], p. 137, have suggested that a non-locality in the direction perpendicular to glide planes would result from considering the double cross-slip of screw dislocations. Various short-range contributions has been theorized also by El Azab [36]. All of these shortrange contributions could be in principle considered in the present framework by introducing the corresponding terms in eq. (14).

\subsection{Fundamental equations}

Consider an area $A$ through which the continuously distributed dislocations of a singlevalued field are piercing. The resulting Burgers vector $\boldsymbol{B}(A, t)$ of all the dislocations that pierce through the area $A$ is

$$
\boldsymbol{B}=\int_{A}(\boldsymbol{b} \otimes \rho \boldsymbol{\xi}) \mathrm{d} \boldsymbol{a}
$$

where $\mathrm{d} \boldsymbol{a}$ is an oriented element of the area $A$. We note in passing the relation of the tensor $\boldsymbol{b} \otimes \rho \boldsymbol{\xi}$ to the tensor $\boldsymbol{\alpha}$, cf. eqs. (1) and (3), as well as the relation of eq. (16) to eq. (10) of the classical theory. On assuming the area $A$ to be fixed in space (Eulerian description), the rate of change in the resulting Burgers vector becomes

$$
\frac{\partial \boldsymbol{B}}{\partial t}=\int_{A} \frac{\partial}{\partial t}(\boldsymbol{b} \otimes \rho \boldsymbol{\xi}) \mathrm{d} \boldsymbol{a} .
$$

This rate of change is caused by dislocations that move with the velocity $\boldsymbol{V}(\boldsymbol{x}, t)$ across the boundary $C$ of the area $A$. It can be determined as follows. With its magnitude corresponding to the hatched area in Fig. 3, [Insert Fig. 3] the vector $\mathrm{d} \boldsymbol{l} \times \boldsymbol{V}$, where $\mathrm{d} \boldsymbol{l}$ is a line element of the boundary $C$, and $\boldsymbol{V}=V \boldsymbol{\nu}$ is the glide velocity, accounts for the velocity component perpendicular to the boundary. The inflow of the oriented dislocation 
lines distributed continuously with the density $\rho \boldsymbol{\xi}$ through the line element $\mathrm{d} \boldsymbol{l}$ is thus determined by

$$
\rho \boldsymbol{\xi} \cdot(\mathrm{d} \boldsymbol{l} \times \boldsymbol{V})=\mathrm{d} \boldsymbol{l} \cdot(\boldsymbol{V} \times \rho \boldsymbol{\xi})=-(\rho \boldsymbol{\xi} \times \boldsymbol{V}) \cdot \mathrm{d} \boldsymbol{l} .
$$

The flow of Burgers vector through the element of the boundary is $-(\boldsymbol{b} \otimes \rho \boldsymbol{\xi} \times \boldsymbol{V}) \mathrm{d} \boldsymbol{l}$. Accordingly, the rate of change in the resulting Burgers vector of the dislocations piercing through the area $A$ which is due to dislocations flowing across its boundary $C$ is

$$
\frac{\partial \boldsymbol{B}}{\partial t}=-\oint_{C}(\boldsymbol{b} \otimes \rho \boldsymbol{\xi} \times \boldsymbol{V}) \mathrm{d} \boldsymbol{l}=-\int_{A} \operatorname{curl}(\boldsymbol{b} \otimes \rho \boldsymbol{\xi} \times \boldsymbol{V}) \mathrm{d} \boldsymbol{a},
$$

where Stoke's theorem has been used. Since the area $A$ is arbitrary, a field equation of evolution follows from eqs. (17) and (18),

$$
\frac{\partial}{\partial t}(\boldsymbol{b} \otimes \rho \boldsymbol{\xi})=-\operatorname{curl}(\boldsymbol{b} \otimes \rho \boldsymbol{\xi} \times \boldsymbol{V}) .
$$

Relation (19) is the fundamental equation that governs the evolution of the scalar density $\rho(\boldsymbol{x}, t)$ and orientation $\boldsymbol{\xi}(\boldsymbol{x}, t)$ of a single-valued dislocation field. As for the physical interpretation of the right-hand side of eq. (19), we note that in view of eq. (12), the term

$$
\rho \boldsymbol{\xi} \times \boldsymbol{V}=\rho V \boldsymbol{\xi} \times \boldsymbol{\nu}=\rho V \boldsymbol{m},
$$

is the local rate of change in the area per volume swept in the glide planes $\boldsymbol{m}$ by the dislocations with density $\rho \boldsymbol{\xi}\left[\mathrm{m} / \mathrm{m}^{3}\right]$ and glide velocity $\boldsymbol{V}=V \boldsymbol{\nu}[\mathrm{m} / \mathrm{s}]$. Accordingly, the second-order tensor $\boldsymbol{b} \otimes \rho \boldsymbol{\xi} \times \boldsymbol{V}$ has the meaning of the plastic slip rate caused by the single-valued dislocation field on the corresponding slip system,

$$
b \boldsymbol{s} \otimes \rho \boldsymbol{\xi} \times V \boldsymbol{\nu}=b \rho V \boldsymbol{s} \otimes \boldsymbol{m}=\dot{\gamma}^{\mathrm{p}} \boldsymbol{s} \otimes \boldsymbol{m} .
$$

This accords with the crystal plasticity $[42,43]$, as well as with the Orowan equation,

$$
\dot{\gamma}^{\mathrm{p}}=b \rho V
$$

Let us note that the Orowan equation (22) represents the rate of change in plastic slip at a fixed position in space $\boldsymbol{x}$. We write more precisely

$$
\frac{\partial \gamma^{\mathrm{p}}}{\partial t}=b \rho V
$$

where $\partial / \partial t$ is the spatial (Eulerian) time derivative. This detail will become important later in Sec. 4.3.

In addition to eq. (19), an evolving dislocation field must fulfil the condition of conservation of Burgers vector which ensures that dislocations cannot end within the crystal. To derive this condition, consider a fixed but otherwise arbitrary volume $\Omega$. Since dislocations cannot end within this volume, the resulting Burgers vector $\boldsymbol{B}(\partial \Omega, t)$ of all 
the dislocations that pierce through its boundary (closed surface) $\partial \Omega$, cf. eq. (16), must vanish,

$$
\boldsymbol{B}=\int_{\partial \Omega}(\boldsymbol{b} \otimes \rho \boldsymbol{\xi}) \mathrm{d} \boldsymbol{a}=\mathbf{0} .
$$

Applying Gauss' theorem to eq. (24), we get

$$
\int_{\partial \Omega}(\boldsymbol{b} \otimes \rho \boldsymbol{\xi}) \mathrm{d} \boldsymbol{a}=\int_{\Omega} \operatorname{div}(\boldsymbol{b} \otimes \rho \boldsymbol{\xi}) \mathrm{d} \Omega=\mathbf{0} .
$$

This yields the field equation,

$$
\operatorname{div}(\boldsymbol{b} \otimes \rho \boldsymbol{\xi})=\mathbf{0},
$$

which represents the local form of the law of conservation of Burgers vector in a medium.

The equations derived above have their counterparts in the classical continuum theory of dislocations [16, 17, 18, 19, 20, 21]. Assuming that the total dislocation content of a crystal consists of several single-valued dislocation fields on different slip systems, the dislocation density tensor $\boldsymbol{\alpha}(\boldsymbol{x}, t)$ of the classical theory, eq. (1), can be obtained as a sum over all the tensors $\boldsymbol{b} \otimes \rho \boldsymbol{\xi}$, eq. (16), corresponding to the individual single-valued fields. Similarly, the total scalar density $\varrho(\boldsymbol{x}, t)$ is the sum of the scalar densities $\rho(\boldsymbol{x}, t)$ of the individual single-valued fields. The rate of plastic distortion $\partial \boldsymbol{\beta}^{\mathrm{p}} / \partial t$ results as a sum of the contributions $\dot{\gamma}^{\mathrm{p}} \boldsymbol{s} \otimes \boldsymbol{m}$, eq. (21), from the individual single-valued fields. Then, eq. (19) yields the fundamental equation of the continuum theory of moving dislocations,

$$
\frac{\partial \boldsymbol{\alpha}}{\partial t}=-\operatorname{curl} \frac{\partial \boldsymbol{\beta}^{\mathrm{p}}}{\partial t}
$$

whereas condition (26) becomes the standard condition of conservation of Burgers vector,

$$
\operatorname{div} \boldsymbol{\alpha}=\mathbf{0}
$$

As discussed in Sec. 2.2, the classical continuum theory of dislocations does not enable us to derive the plastic strain rate from the density and velocity of the continuously distributed dislocations. Moreover, the dislocation curvature $\kappa$, eq. (13), cannot be determined within the framework of the classical theory. However, all this is possible in the framework of the theory of single-valued dislocation fields to which we return in the following.

\section{Evolution of dislocation density and orientation}

Treating the single-valued dislocation fields separately, we will be able to determine the evolution of the scalar density $\rho(\boldsymbol{x}, t)$ and orientation $\boldsymbol{\xi}(\boldsymbol{x}, t)$ of a single-valued dislocation field, as well as the plastic slip rate $\dot{\gamma}^{\mathrm{p}}(\boldsymbol{x}, t)$ resulting from this evolution. In Sections 4.1 and 4.2, we keep on using the spatial (Eulerian) description. In Sections 4.3 and 4.4, the so-called dislocation-Lagrangian approach will be utilized. 


\subsection{Eulerian form of the evolution equations}

We want to employ eq. (19) as an equation governing the evolution of the density $\rho(\boldsymbol{x}, t)$ and orientation $\boldsymbol{\xi}(\boldsymbol{x}, t)$ of a single-valued dislocation field. To this end, we have to prove first that this evolution equation keeps on conserving the Burgers vector, provided, the initial dislocation configuration fulfills the conservation condition (26).

Lemma 4.1.1 Let the evolution of density $\rho(\boldsymbol{x}, t)$ and orientation $\boldsymbol{\xi}(\boldsymbol{x}, t)$ of a singlevalued dislocation field be governed by eq. (19) for $t \geq t_{0}$. Let these fields in time $t=t_{0}$ satisfy eq. (26) (initial condition). Then eq. (26) holds for all $t \geq t_{0}$.

Proof. By taking divergence of eq. (19), changing the order of differentiation, and noting the identity 'div curl $=0$ ', we get

$$
\frac{\partial}{\partial t} \operatorname{div}(\boldsymbol{b} \otimes \rho \boldsymbol{\xi})=\mathbf{0}
$$

Accordingly, since eq. (26) holds for $t=t_{0}$, it holds also for all $t \geq t_{0}$.

In the following, we derive the individual evolution equations for the density $\rho(\boldsymbol{x}, t)$ and orientation $\boldsymbol{\xi}(\boldsymbol{x}, t)$. Utilizing eq. (20) and noting that the vectors $\boldsymbol{s}$ and $\boldsymbol{m}$ are constant in space, we rewrite the right-hand side of eq. (19),

$$
-\operatorname{curl}(b \rho V \boldsymbol{s} \otimes \boldsymbol{m})=-\boldsymbol{s} \otimes(\operatorname{grad}(b \rho V) \times \boldsymbol{m})=-\boldsymbol{s} \otimes(\operatorname{grad}(b \rho V) \times(\boldsymbol{\xi} \times \boldsymbol{\nu})) .
$$

Utilizing the identity ' $\boldsymbol{u} \times(\boldsymbol{v} \times \boldsymbol{w})=(\boldsymbol{u} \cdot \boldsymbol{w}) \boldsymbol{v}-(\boldsymbol{u} \cdot \boldsymbol{v}) \boldsymbol{w}$ ', we decompose the tensor equation (19) in the glide plane locally into directions tangential and normal to the dislocation lines,

$$
\begin{aligned}
\frac{\partial b \rho}{\partial t} \boldsymbol{s} \otimes \boldsymbol{\xi} & =-(\operatorname{grad}(b \rho V) \cdot \boldsymbol{\nu}) \boldsymbol{s} \otimes \boldsymbol{\xi} \\
b \rho \boldsymbol{s} \otimes \frac{\partial \boldsymbol{\xi}}{\partial t} & =(\operatorname{grad}(b \rho V) \cdot \boldsymbol{\xi}) \boldsymbol{s} \otimes \boldsymbol{\nu}
\end{aligned}
$$

We note in passing that, since $b$ is a constant in a single-valued dislocation field, it cancels out in the above equations. However, to be consistent with the continuum limit, eq. (5), we treat the product $b \rho(\boldsymbol{x}, t)$ as a unit. Moreover, we preserve thereby the expression $b \rho V$ which has the physical meaning of plastic slip rate, $b \rho V=\dot{\gamma}^{\mathrm{p}}$, eq. (22). Equation (30) yields a scalar evolution equation for the dislocation density,

$$
\frac{\partial b \rho}{\partial t}=-\operatorname{grad}(b \rho V) \cdot \boldsymbol{\nu} \equiv-\partial_{\boldsymbol{\nu}}(b \rho V)
$$

which shows that the rate of change in the scalar density $\rho$ of a single-valued dislocation field equals the derivative in the normal direction $\boldsymbol{\nu}$ of the rate of change in the area per 
volume $\rho V$ slipped by the dislocations. It is instructive to rewrite eq. (32) in the standard form of a continuity equation, namely,

$$
\frac{\partial b \rho}{\partial t}+\operatorname{div}(b \rho \boldsymbol{V})=-b \rho V \kappa .
$$

Accounting for the change in length of the curved gliding dislocations, the right-hand side of eq. (33) shows that curved dislocation segments do not behave as standard particles (for which the right-hand side would vanish). Since no production of 'new' dislocations is considered, this change in length accounts for the physical notion of curved dislocation lines that do not disintegrate in the course of the evolution, cf. the example in Sec. 4.2 below.

To be able to analyze eq. (31), we resort to a system of coordinates $x_{1} O x_{2}$ in the glide plane. Then, by introducing a scalar field of the angle $\vartheta(\boldsymbol{x}, t)$ between the $x_{1}$ axis and tangent $\boldsymbol{\xi}(\boldsymbol{x}, t)$, the fields of tangent and normal vectors to dislocation lines can be expressed as

$$
\begin{aligned}
\boldsymbol{\xi} & =(\cos \vartheta, \sin \vartheta), \\
\boldsymbol{\nu} & =(-\sin \vartheta, \cos \vartheta) .
\end{aligned}
$$

Now we see that $\partial \boldsymbol{\xi} / \partial t=\partial \vartheta / \partial t \boldsymbol{\nu}$ and we infer from eq. (31) that

$$
b \rho \frac{\partial \vartheta}{\partial t}=\operatorname{grad}(b \rho V) \cdot \boldsymbol{\xi} \equiv \partial_{\boldsymbol{\xi}}(b \rho V) .
$$

In order to get a consistent system of equations, we deduce from the conservation equation for Burgers vector $(26)$ in the form $\operatorname{div}(\boldsymbol{s} \otimes b \rho \boldsymbol{\xi})=\mathbf{0}$ the condition for the vector $b \rho \boldsymbol{\xi}$ of the single-valued field being solenoidal,

$$
\operatorname{div}(b \rho \boldsymbol{\xi})=0 .
$$

The physical meaning of condition (36) is that dislocations cannot begin or end in the medium.

The coupled evolution of the dislocation density $\rho(\boldsymbol{x}, t)$ and orientation $\vartheta(\boldsymbol{x}, t)$ is governed by eqs. (32) and (35), whereas condition (36) must be imposed on the initial dislocation configuration. The coupling of the density and orientation in these evolution equations then guarantees the continuing conservation of Burgers vector, eq. (29).

Another possibility to account for the evolution of a single-valued dislocation field is to introduce condition (36) in eq. (35) explicitly. The right-hand side of eq. (35) can be rewritten as

$$
\operatorname{grad}(b \rho V) \cdot \boldsymbol{\xi}=\operatorname{div}(b \rho V \boldsymbol{\xi})-b \rho V \operatorname{div} \boldsymbol{\xi}=\operatorname{grad} V \cdot b \rho \boldsymbol{\xi}+V \operatorname{div}(b \rho \boldsymbol{\xi})-b \rho V \operatorname{div} \boldsymbol{\xi} .
$$

Then, eq. (35) together with condition (36) yields the evolution equation for the dislocation orientation $\vartheta(\boldsymbol{x}, t)$,

$$
\frac{\partial \vartheta}{\partial t}=\operatorname{grad} V \cdot \boldsymbol{\xi}-V \operatorname{div} \boldsymbol{\xi} .
$$


The first term on the right-hand side of the (Eulerian) evolution equation (37) represents the net rate of change in orientation of a dislocation segment due to the gradient of the glide velocity along it, the second term is a convective one. Roughly speaking, it accounts for dislocation segments of varying orientation flowing with the velocity $V$ in the considered fixed elementary volume positioned at $\boldsymbol{x}$ from its neighborhood.

Now the opportunity arises to compute the evolution of the orientation from eq. (37) and the evolution of the density from this orientation and condition (36) itself, without using eq. (32) for the density at all. It should be checked, however, that this possibility is admissible, i.e. that the 'correct' evolution of the density consistent with the (now unused) eq. (32) results.

Lemma 4.1.2 Let the evolution of the orientation $\vartheta(\boldsymbol{x}, t)$ be governed by eq. (37) for $t \geq t_{0}$. Let the current density $\rho(\boldsymbol{x}, t)$ be determined from this orientation and condition (36) itself for all $t \geq t_{0}$. Then eq. (32) is fulfilled implicitly for all $t \geq t_{0}$.

Proof. Since condition (36) is fulfilled for all $t \geq t_{0}$, it follows that $\partial \operatorname{div}(b \rho \boldsymbol{\xi}) / \partial t=0$. Changing the order of time and space differentiation, we get

$$
\operatorname{div} \frac{\partial(b \rho \boldsymbol{\xi})}{\partial t}=\operatorname{div}\left(\frac{\partial b \rho}{\partial t} \boldsymbol{\xi}+b \rho \frac{\partial \vartheta}{\partial t} \boldsymbol{\nu}\right)=0
$$

In view of the identity 'div curl $=0$ ', the term in brackets can be written as a curl of a vector $a \boldsymbol{m}$ perpendicular to the plane spanned by the vectors $\boldsymbol{\xi}$ and $\boldsymbol{\nu}$,

$$
\frac{\partial \rho}{\partial t} \boldsymbol{\xi}+\rho \frac{\partial \vartheta}{\partial t} \boldsymbol{\nu}=\operatorname{curl}(a \boldsymbol{m})
$$

where the magnitude $a$ of the vector $a \boldsymbol{m}$ is unknown. The evolution of the orientation $\partial \vartheta / \partial t$ is governed by eq. (37). Utilizing condition (36) and following the derivation that has lead to eq. (37) backwards, we come to eq. (35) which we use in the left-hand side of the above equation. As for the right-hand side of this equation, we apply the idea that has lead us from eq. (19) to eqs. (30) and (31) to the vector $\boldsymbol{a m}$. We get

$$
\begin{aligned}
\frac{\partial \rho}{\partial t} \boldsymbol{\xi} & =(\operatorname{grad}(a) \cdot \boldsymbol{\nu}) \boldsymbol{\xi}, \\
(\operatorname{grad}(\rho V) \cdot \boldsymbol{\xi}) \boldsymbol{\nu} & =-(\operatorname{grad}(a) \cdot \boldsymbol{\xi}) \boldsymbol{\nu} .
\end{aligned}
$$

From the latter equation we get $a=-\rho V$. Substituting this into the former equation, we arrive at eq. (32).

We conclude that the evolution of density and orientation can be computed in two ways. Either by using the pair of eqs. (32) and (35), whereas the conservation of Burgers vector (36) plays the role of an initial condition and is accounted for implicitly in the course of the evolution, cf. Lemma 4.1.1. Or, alternatively, using eq. (37) for the orientation evolution together with condition (36) for the density which leads to the same result, cf. Lemma 4.1.2. The practical importance of this result will become clear in Sec. 5 below where the theory is specialized to one spatial dimension. 


\subsection{Expansion of concentric dislocation loops (Eulerian description)}

The evolution equations will now be applied to a simple example of concentric circular dislocation loops expanding in a constant and homogeneous stress field $\bar{\tau}$. We can think of the loops as having been produced by a Frank-Read source at the center, whereas we do not take care of the details of the source operation. Since the loops are concentric, the dislocation field is single-valued. By means of this example, we want to demonstrate that the present evolution equations correctly reflect the increase in length of the expanding loops. Moreover, we want to test numerical methods that can be used to solve the evolution equations.

Polar coordinates $r, \theta$ are introduced, with the corresponding unit base vectors $\boldsymbol{e}_{r}, \boldsymbol{e}_{\theta}$, Fig. 4. [Insert Fig. 4] The initial configuration in time $t=t_{0}$ consists of concentric circular dislocation loops distributed continuously in the slip plane with a density $\rho\left(r, t_{0}\right)$ such that

$$
\begin{array}{ll}
\rho>0 & \text { for } \quad r \in\left[R_{1}, R_{2}\right] \\
\rho=0 & \text { otherwise. }
\end{array}
$$

In polar coordinates, the vectors tangent and normal to the dislocations, eq. (34), become

$$
\begin{aligned}
& \boldsymbol{\xi}=\boldsymbol{e}_{\theta}, \\
& \boldsymbol{\nu}=\boldsymbol{e}_{r} .
\end{aligned}
$$

In the following, we neglect the yield stress $\hat{\tau}$, and assume that $\bar{\tau}>T /\left(b R_{1}\right)$. Then, the yield condition (14) reduces to the equation of motion in the form,

$$
B V=\bar{\tau} b-T \frac{1}{r}
$$

where $-1 / r=\kappa(r)$ is the curvature of the expanding loops. The line tension $T$ alone thus provides the resistance to the expansion of the dislocation loops in this example. It must be pointed out that these simplifying assumptions have been made to make the example transparent as well as to get an analytical solution, cf. Appendix A, that serves to check the accuracy of the various numerical implementations. This example aims at showing the consistency and mathematical properties of the present form of continuum dislocation kinematics, especially the increase in length of the continuously distributed expanding dislocation loops. Whereas the problem of an individual expanding or shrinking dislocation loop is a standard one and treated by many researchers in the past, cf. eg. the recent work [30], it becomes nontrivial in a continuum description. This example shows that the present theory can treat this problem correctly. In general, it is of course possible to keep a non-zero yield stress $\hat{\tau}$ as well as the full yield condition (14) in this example. Then, one would be able to simulate an expanding, stationary or shrinking loop density in a field of forest 
dislocations represented by the yield stress $\hat{\tau}$, cf. eq. (11). Admittedly, the problem of annihilation (when the loops shrink to zero size) is not treated in the present approach.

For the reader's convenience, we recall the form of the operators grad and div in polar coordinates,

$$
\begin{aligned}
\operatorname{grad} a & =\frac{\partial a}{\partial r} \boldsymbol{e}_{r}+\frac{1}{r} \frac{\partial a}{\partial \theta} \boldsymbol{e}_{\theta}, \\
\operatorname{div} \boldsymbol{a} & =\frac{1}{r} \frac{\partial\left(r a_{r}\right)}{\partial r}+\frac{1}{r} \frac{\partial a_{\theta}}{\partial \theta},
\end{aligned}
$$

where $a$ is a scalar and $\boldsymbol{a}=a_{r} \boldsymbol{e}_{r}+a_{\theta} \boldsymbol{e}_{\theta}$ a vector. Now we check the validity of the conservation condition (36). In view of eq. $(39)_{1}$ and $(41)_{2}$ we get

$$
\operatorname{div}(\rho \boldsymbol{\xi})=\frac{1}{r} \frac{\partial \rho(r)}{\partial \theta}=0
$$

as expected in the case of closed loops. As for the evolution of the orientation $\vartheta(\theta, t)$, by applying eq. (35) and (41) $)_{1}$ we get

$$
\rho \frac{\partial \vartheta}{\partial t}=\frac{\partial(\rho V)}{\partial r} \boldsymbol{e}_{r} \cdot \boldsymbol{e}_{\theta}=0
$$

Since $\rho>0$, eq. (38), this means that $\partial \vartheta / \partial t=0$, as expected. It can be checked that the same result follows from application of eq. (37). Finally, the evolution of the density $\rho(r, t)$ can be computed from eq. (32) as

$$
\frac{\partial \rho}{\partial t}=-\frac{\partial(\rho V)}{\partial r} \boldsymbol{e}_{r} \cdot \boldsymbol{e}_{r}=-\frac{\partial(\rho V)}{\partial r} .
$$

Equation (44) together with the initial condition (38) constitutes an initial-value problem for the density $\rho(r, t)$, whereas the velocity $V(r, t)$ is given by the algebraic eq. (40). For simplicity, we consider a step-like initial dislocation distribution, $\rho=$ const. for $r \in$ $\left[R_{1}, R_{2}\right]$. Since the interest here is in the qualitative behavior of the evolution equation, we do not list the numerical values of the input parameters used.

The Cauchy problem of the density evolution will be solved analytically first. The analytical solution will serve to check the accuracy of later numerical computations. Starting point for the analytical solution of the Cauchy problem is eq. (44) in the form

$$
\frac{\partial \rho}{\partial t}+V \frac{\partial \rho}{\partial r}=-\rho \frac{\partial V}{\partial r}
$$

which reveals its convective character (see the second term on the left-hand side). The key to the solution of this equation are the characteristic curves in the $r$ - $t$ plane that fulfill relation

$$
\frac{\mathrm{d} r}{\mathrm{~d} t}=V
$$


The analytical solution to the initial-value problem is given in terms of the Lambert $W$ function in Appendix A and plotted in Fig 5. [Insert Fig. 5] It will be used to check the correctness of the numerical computations reported below.

We get a better insight in the physical meaning of the density evolution by considering it in the form of the continuity eq. (33). In the present case, eq. (33) yields

$$
\frac{\partial \rho}{\partial t}+\frac{1}{r} \frac{\partial(r \rho V)}{\partial r}=\rho V \frac{1}{r}
$$

This can be rewritten as

$$
\frac{\partial \rho}{\partial t}=-\rho V \frac{1}{r}-\frac{\partial(\rho V)}{\partial r}+\rho V \frac{1}{r}
$$

The first term on the right-hand side of eq. (48) represents the decrease of the density that would be caused by expansion of the loops consisting of a constant number of elementary segments of unchanging length. Such an expansion, corresponding to dislocation segments that behave as standard particles, would lead to disintegration of the loops, cf. Zaiser and El-Azab [44]. The present theory requires that this term be compensated by the last term on the right-hand side of eq. (48) which accounts for the increasing length of the expanding segments. The second term on the right-hand side is the correct rate of change in the density that accords with eq. (44). It should be pointed out that the disintegration of the loops that would result from the missing last term on the right-hand side of eq. (48) could not be directly observed in the continuum description. However, the disintegration can be deduced from the evolution of the total length $L(t)$ of the loops that can be computed $\mathrm{as}^{3}$

$$
L(t)=\int_{0}^{\infty} 2 \pi r \rho(r, t) \mathrm{d} r .
$$

The disintegration would be reflected by an unchanging total length, $L(t)=L\left(t_{0}\right)$ for all $t \geq t_{0}$. The total length of the expanding loops that do not disintegrate must steadily increase, $L\left(t_{2}\right)>L\left(t_{1}\right)$ for all $t_{2}>t_{1} \geq t_{0}$.

The output of the present continuum model of evolving dislocation fields is the plastic slip rate, eq. (21). It should be noted that we still solve a coupled continuum mechanics dislocation dynamics problem in the sense of Fig. 1. Although the continuum mechanics part is trivial in the current example, the coupling to crystal plasticity still persists. In the present example, the plastic slip rate becomes

$$
\dot{\gamma}^{\mathrm{p}}(r, t)=b \rho(r, t) V(r, t) .
$$

It can be time-integrated to yield the plastic slip $\gamma^{\mathrm{P}}(r, t)$. To be able to solve this initial value problem parallel with the solution of eq. (44), we need the initial condition $\gamma^{\mathrm{p}}\left(r, t_{0}\right)$.

\footnotetext{
${ }^{3}$ To be consistent with other parts of the text, we keep the meaning of $\rho\left[\mathrm{m}^{-2}\right]$ as a volume density, although the dislocations in this example are distributed in a plane. As a consequence, the 'length' computed in eq. (49), being reduced by a unit length measured in the $\boldsymbol{m}$ direction, is a dimensionless quantity.
} 
Assuming, the loops have expanded from the center in time $t<t_{0}$, the initial plastic slip can be determined from the initial dislocation distribution (38) as

$$
\gamma^{\mathrm{p}}\left(r, t_{0}\right)=\int_{r}^{\infty} b \rho\left(\tilde{r}, t_{0}\right) \mathrm{d} \tilde{r}
$$

Roughly speaking, by applying eq. (51), we count the loops that have passed the point $r$. The knowledge of the value of plastic slip in the course of the evolution, $\gamma^{\mathrm{p}}(r, t)$, $t>t_{0}$, gives us the possibility to check whether the resulting evolution is reasonable: all the points $r$ that have been passed by all the expanding loops at any time must have experienced the same plastic slip. On the other hand, if the loops disintegrate, the plastic slip in the points farther from the center that were passed by all the loops at later times would be smaller.

The Cauchy problem consisting of the coupled differential equations (44) and (50) with initial conditions (38) and (51) has been solved numerically. We have used standard Runge-Kutta forward integration with adaptive step size in time and backward (i.e. upwind) differentiation in space on a fixed grid with 10000 nodes. When employing only e.g. 1000 nodes, we observe a more pronounced blurring of the edges of the resulting curves representing the evolving density, Fig. 6. [Insert Fig. 6] The high resolution is thus needed especially for the differentiation of the step-like edges of the density. In fact, the step-like initial configuration considered is the 'worst case' for the numerical computation. Note that a forward or central differentiation in space fails for the convective equation solved. The evolution of the density and plastic slip of the expanding loops is shown in the left part of Fig. 6. For comparison, we have solved the same problem using eq. (47) with its right-hand side set to zero. This corresponds to loops consisting of a constant number of segments of unchanging length that disintegrate during the evolution. The computation has certified the expectation expressed above, namely, that the continuum model yields in this case a too low and still decreasing density as well as a decreasing plastic slip, see the right part of Fig. 6. We have checked that in this latter case, the total length of the dislocations computed from eq. (49) remains constant during the evolution which is inconsistent with the picture of expanding dislocation loops of increasing length.

The example indicates that the evolution equations (32) and (33) for the dislocation density $\rho(\boldsymbol{x}, t)$ correctly account for the changing length of continuously distributed gliding curved dislocations.

\subsection{Dislocation-Lagrangian form of the evolution equations}

The evolution equations for the density and orientation of a single-valued dislocation field have been derived in the Eulerian (spatial) form that accounts for the evolution of these fields at fixed positions $\boldsymbol{x}$ in space. In the dislocation-Lagrangian description, the point of observation moves along with a dislocation segment. We can imagine it as a virtual 
particle $X$ moving with the dislocation velocity through the material and carrying along the information on the dislocation orientation, density, and, possibly, plastic slip. Let us assign a position vector $\boldsymbol{\varphi}(X, t)$ to such a particle, so that $\boldsymbol{x}=\boldsymbol{\varphi}(X, t)$ is the position in space of the particle $X$ at time $t$. The label $X$ denoting the particle can be interpreted as the position of this particle in a reference configuration. The rate of change in the position $\varphi(X, t)$ of the particle $X$ (the dislocation-Lagrangian picture) equals the velocity of the dislocation field $\boldsymbol{V}(\boldsymbol{x}, t)$ at the current position $\boldsymbol{x}$ of the particle (the Eulerian picture),

$$
\frac{\mathrm{d} \boldsymbol{\varphi}(X, t)}{\mathrm{d} t}=\boldsymbol{V}(\boldsymbol{x}, t) .
$$

In the dislocation-Lagrangian description, the net evolution of the density and orientation of the dislocation fields is separated from the convective effects related to the dislocation velocity. For a scalar field $a(\boldsymbol{x}, t)$, the dislocation-Lagrangian time derivative $\mathrm{d} / \mathrm{d} t$ is related to the Eulerian (spatial) time derivative $\partial / \partial t$ through the velocity $\boldsymbol{V}(\boldsymbol{x}, t)$ of the dislocation field,

$$
\frac{\mathrm{d} a}{\mathrm{~d} t}=\frac{\partial a}{\partial t}+\operatorname{grad} a \cdot \boldsymbol{V} .
$$

The term $\operatorname{grad} a \cdot \boldsymbol{V} \equiv V \partial_{\boldsymbol{\nu}} a$, that is the derivative in the direction of the velocity multiplied by the velocity magnitude, is known as the convective derivative of the respective field. Thus in the dislocation-Lagrangian description, moving along with dislocation segments, we are able to monitor their net change in length and orientation.

Utilizing relation (53), Sedláček et al. [23] derived the dislocation-Lagrangian form of the evolution equations for the dislocation density and orientation. Here we summarize these evolution equations and derive additionally the dislocation-Lagrangian form of the plastic slip rate (23). Evolution eq. (32) for the scalar density $\rho(\boldsymbol{x}, t)$ becomes

$$
\frac{\mathrm{d} b \rho}{\mathrm{d} t}=-b \rho \operatorname{grad} V \cdot \boldsymbol{\nu} \equiv-b \rho \partial_{\nu} V .
$$

This means, the dislocation density in a flowing volume element changes with the gradient of the velocity magnitude in the velocity direction (i.e. perpendicular to dislocations). The latter evolution equation still accounts for the change in length of the curved gliding dislocations, as it is seen in the right-hand side of the dislocation-Lagrangian form of the continuity eq. (33), that is

$$
\frac{\mathrm{d} b \rho}{\mathrm{d} t}+b \rho \operatorname{div} \boldsymbol{V}=-b \rho V \kappa
$$

The dislocation-Lagrangian form of evolution eq. (35) for the orientation $\vartheta(\boldsymbol{x}, t)$ is

$$
b \rho \frac{\mathrm{d} \vartheta}{\mathrm{d} t}=b \rho V \operatorname{div} \boldsymbol{\xi}+\operatorname{grad}(b \rho V) \cdot \boldsymbol{\xi}
$$

where we have utilized relation $\operatorname{grad} \vartheta \cdot \boldsymbol{\nu}=\operatorname{div} \boldsymbol{\xi}$. Upon introducing condition (36) in eq. (56), the dislocation-Lagrangian form of evolution eq. (37) results,

$$
\frac{\mathrm{d} \vartheta}{\mathrm{d} t}=\operatorname{grad} V \cdot \boldsymbol{\xi} \equiv \partial_{\boldsymbol{\xi}} V
$$


cf. the derivation of eq. (37) itself. Equation (57) describes the net rate of rotation of gliding dislocation segments. The dislocation-Lagrangian plastic slip rate follows from the Orowan eq. (23) and relation (53) as

$$
\frac{\mathrm{d} \gamma^{\mathrm{p}}}{\mathrm{d} t}=b \rho V+\operatorname{grad} \gamma^{\mathrm{p}} \cdot \boldsymbol{V} .
$$

In the next Section, we apply the evolution equation in the dislocation-Lagrangian form to the example of expanding dislocation loops that was treated in the Eulerian description in Sec. 4.2 and compare the results.

\subsection{Expansion of concentric dislocation loops (dislocation-Lagrangian description)}

Utilizing the dislocation-Lagrangian formalism, we solve the problem of expanding circular dislocation loops that has been posed in Sec. 4.2. The relevant evolution equations written in polar coordinates are as follows. Equation (54) for the density becomes

$$
\frac{\mathrm{d} \rho}{\mathrm{d} t}=-\rho \frac{\partial V}{\partial r}
$$

This equation represents the net rate of change in the dislocation density caused by the gradient of the velocity in the direction of motion in a flowing volume. It corresponds to eq. (45) with the convective term eliminated. The dislocation-Lagrangian rate of plastic slip follows from eq. (58) as

$$
\frac{\mathrm{d} \gamma^{\mathrm{p}}}{\mathrm{d} t}=b \rho V+\frac{\partial \gamma^{\mathrm{p}}}{\partial r} V
$$

We recognize the standard Orowan relation in the first term on the right-hand side and the convective derivative in the second term. The dislocation flow is described by eq. (52) which becomes

$$
\frac{\mathrm{d} \varphi_{r}}{\mathrm{~d} t}=V .
$$

This equation determines the evolving radial position $\varphi_{r}(r, t)$ of the virtual particles that carry the information on the density and plastic slip (the orientation does not change in this example, see Sec.4.2). The initial condition $\varphi_{r}\left(r, t_{0}\right), r \in\left[R_{1}, R_{2}\right]$, corresponds to the initial position of the nodes for the numerical solution of the problem. Note that with $\varphi_{r}(r, t)=r(t)$, eq. (61) is equivalent to the equation (46) of the characteristic curve. This means that the dislocation-Lagrangian derivative, eq. (59), describes the time rate of change in the density along the characteristics defined by eq. (46), cf. Fig. 5 .

We summarize the differences between the dislocation-Lagrangian and Eulerian approaches. Instead of two coupled equations, we have to solve three. The unknown functions of the Cauchy problem in the dislocation-Lagrangian form are the density $\rho(r, t)$ with the initial condition (38), plastic slip $\gamma^{\mathrm{p}}(r, t)$ with the initial condition given by eq. (51), and the 
radial position $\varphi_{r}(r, t)$ of the virtual particles carrying the information on the dislocation density and plastic slip, with the initial condition $\varphi_{r}\left(r, t_{0}\right), r \in\left[R_{1}, R_{2}\right]$. In a numerical implementation, the virtual particles are represented by nodes. Accordingly, we need to introduce nodes only in the interval $r \in\left[R_{1}, R_{2}\right]$. They will then move along with the expanding loops, eq. (61), that means along the characteristic curves (46). In this sense, this approach can be seen as a dislocation-Lagrangian particle-tracking method. Moreover, we do not need to differentiate the step-like density in the convective term in the Eulerian eq. (54), cf. the dislocation-Lagrangian eq. (59), which dramatically reduces the computational requirements on the numerical method.

For the numerical computation, we have used a grid of 100 dynamically self-adjusting nodes, the first and last one being placed off the interval $\left[R_{1}, R_{2}\right]$ to enable the differentiation in the boundary points themselves. (Reasonable results have been obtained using as few as 10 nodes as well.) We use central differentiation of the velocity and upwind differentiation of the plastic slip. The CPU time required is only a small fraction of the one needed in the Eulerian approach, whereas the results of the dislocation-Lagrangian method are much more accurate. Especially, we do not observe any blurring of the sharp edges in the density distribution, Fig. 7. [Insert Fig. 7] The numerical solution is in fact indistinguishable from the exact analytical solution shown in Fig. 5. With an increasing number of nodes, the results based on the Eulerian approach converge to the results of the Lagrangian approach (and so to the exact solution). This indicates that, from a theoretical point of view, the Eulerian and dislocation-Lagrangian methods are equivalent, as it should be.

\section{Curved dislocations in one spatial dimension}

The present continuum description of evolving dislocation fields enables us to exploit symmetries of idealized problems to reduce the problem dimensionality. For instance, the dislocation loops expanding in the plastic channel, Fig. 2, can be treated as a spatially one-dimensional problem in the direction across the channel, provided that the loops are distributed continuously and homogeneously along the channel of constant width. In accord with the present theory of single-valued dislocation fields, where the 'upper' and 'lower' halves of the loops are treated separately, only the orientation and density of the dislocation field at a certain position in the direction across the channel is relevant for the evolution of the field. This problem has been described in detail and solved by Sedláček et al. [23]. Problems of this type can occur only in the presence of suitably oriented interfaces that enforce the homogeneity in the remaining directions, cf. Fig. 2. Another example is shown in Fig. 8, [Insert Fig. 8] where the free surface and the interface enforce the homogeneity along the $y$ axis. Moreover, if both the Burgers vector $\boldsymbol{b}$ and the normal vectors $\boldsymbol{m}$ of all slip systems considered are confined to a plane, say, $x O z$, Fig. 8, a plastic strain in that plane results, cf. eq. (21). Then, a plane-strain problem 
of continuum mechanics can be set up to be coupled with the dislocation evolution in the sense of Fig. 1. Even then, the evolution of the dislocation fields can be treated as a one-dimensional problem in the slip direction. With regard to such applications, we specialize the theory of evolving dislocation fields to one spatial dimension.

\subsection{Eulerian evolution equations in $1 \mathrm{D}$}

Motivated by the problem sketched in Fig. 8, we introduce a system of coordinates $x_{1} O x_{2}$ in a glide plane, whereas the Burgers vector is parallel with the $x_{1}$ axis, Fig. 9. An interface, impenetrable for the dislocations, and a free surface, both parallel with the $x_{2}$ axis, are positioned at $x_{1}=0$ and $x_{1}=H$, respectively. Curved dislocations are assumed to be distributed continuously and in the $x_{2}$ direction homogeneously, so that all the field variables are functions of $x_{1}$ only. One representative dislocation in a typical bowed-out configuration is sketched in Fig. 9. [Insert Fig. 9] In accord with the concept of singlevalued dislocation fields, only one half of the dislocation half-loop is considered. Due to the symmetry of the problem, the other half of the loop is a mirror image of the considered one, cf. Fig. 2. The boundary condition for the dislocations at the impenetrable interface is

$$
V=0 \text { for } \quad x_{1}=0 .
$$

The boundary condition at the free surface,

$$
\vartheta=0 \text { for } x_{1}=H
$$

approximates the effect of image forces that tend to orient a dislocation perpendicular to the free surface. Depending on the problem at hand, boundary conditions of this type can be combined at need.

In view of eq. $(34)_{1}$, condition (36) stating that dislocations do not end in the material becomes

$$
\frac{\partial(\rho \cos \vartheta)}{\partial x_{1}}=0 .
$$

From this condition we get by integration,

$$
\rho \cos \vartheta=\bar{\rho}
$$

where the integration constant $\bar{\rho}$ represents a homogeneous density of straight dislocation lines stretched in the $x_{1}$ direction, cf. Fig. 9. It is convenient to consider it as a reference configuration. The current density $\rho\left(x_{1}, t\right)$ thus reflects the increase in length due to the bowing of the dislocations from the reference configuration. Equation (65) can be used conveniently to determine the density, whereas the orientation results from eq. (37) which takes the form

$$
\frac{\partial \vartheta}{\partial t}=\frac{\partial V}{\partial x_{1}} \cos \vartheta-V \frac{\partial \cos \vartheta}{\partial x_{1}} .
$$


We have shown in the proof of Lemma 4.1.2 that this yields the correct density, whereas the evolution eq. (32) for the density need not be solved explicitly. The curvature $\kappa\left(x_{1}, t\right)$ determined from eq. (13) and $(34)_{2}$ as

$$
\kappa=\frac{\partial \sin \vartheta}{\partial x_{1}}
$$

enters the equation of motion (14). The plastic slip rate results from the (Eulerian) Orowan equation (23).

To summarize, in a one-dimensional initial-boundary-value problem for an evolving dislocation field, one has essentially to solve the evolution eq. (66) for dislocation orientation. In the following, we argue that, from the mathematical point of view, this is a transport equation of diffusion-convection type with dominating convection that is known for being difficult to be solved numerically. Typically, solutions of equations of this type possess boundary layers where the derivatives of the solution are very large. The width of these layers is significantly smaller than a reasonable mesh size, so that these layers cannot be properly resolved. This leads to spurious oscillations in the numerical solution in convection-dominated regimes [26]. To analyze the present case, consider eq. (66) in the form

$$
\frac{\partial \vartheta}{\partial t}=\cos \vartheta \frac{\partial V}{\partial x_{1}}+V \sin \vartheta \frac{\partial \vartheta}{\partial x_{1}} .
$$

Upon neglecting the yield stress $\hat{\tau}$ and assuming a homogeneous shear stress $\bar{\tau}$, the dislocation velocity $V$, eq. (14), results in the form,

$$
V=\frac{\bar{\tau} b}{B}+\frac{T \cos \vartheta}{B} \frac{\partial \vartheta}{\partial x_{1}}
$$

where we have utilized the curvature (67). Combining the above two equations, we end up with a non-linear diffusion-convection equation,

$$
\frac{\partial \vartheta}{\partial t}=\frac{T(\cos \vartheta)^{2}}{B} \frac{\partial^{2} \vartheta}{\partial x_{1}^{2}}+\frac{\bar{\tau} b \sin \vartheta}{B} \frac{\partial \vartheta}{\partial x_{1}}
$$

The first term is diffusive, the second one convective. Multiplying the convective coefficient by a length $\Delta x \leq H$, we get a dimensionless ratio of the coefficients of the convective and diffusive terms known as the Péclet number,

$$
\mathrm{Pe}=\frac{\Delta x \bar{\tau} b \sin \vartheta}{T(\cos \vartheta)^{2}}
$$

This number determines whether eq. (68), that is our eq. (66), actually, is locally (i.e. within a particular interval $\Delta x$ ) convection dominated or diffusion dominated [26]. Using order-of-magnitude estimates, $\Delta x \approx 10^{-6}[\mathrm{~m}], \bar{\tau} \approx 10^{7}\left[\mathrm{Nm}^{-2}\right], b \approx 10^{-10}[\mathrm{~m}]$ and $T \approx$ $10^{-9}[\mathrm{~N}]$, we get $\Delta x \bar{\tau} b / T \approx 1$, so that the dislocation orientation $\vartheta(x)$ itself determines the character of the transport equation (68). For slightly bowed-out dislocations, cf. the 
reference configuration of straight dislocation lines, Fig. 9, we have $\vartheta \approx 0$ everywhere, so that $\mathrm{Pe} \ll 1$, eq. (68) thus being diffusion dominated. For dislocations in the typical bowed-out configuration, $\vartheta \rightarrow \pi / 2$ in the vicinity of an impenetrable interface, Fig. 9, yielding $\mathrm{Pe} \gg 1$, so that eq. (68) is strongly convection dominated near the interface.

To support the analysis, we carry out a test computation of a one-dimensional dislocation field of Fig. 9. We start with the reference configuration of straight dislocation lines. A constant shear stress $\bar{\tau}$ is applied in steps of 0.1 of the Orowan stress, $\tau_{\text {Or }}=T / b H$, from $0.1 \tau_{\text {Or }}$ up to $1 \tau_{\text {Or }}$. Equation (66) is repeatedly solved for the orientation $\vartheta(x)$ until an equilibrium configuration is reached in each loading step, i.e. until the dislocation velocity $V(x)$ is negligible. In addition, plastic slip $\gamma^{\mathrm{p}}(x)$ is computed by integrating the Orowan relation (22). We have used central-differentiation on a regular mesh of 13 nodes and an implicit time integration with step-size control. The results of the numerical computation have been compared with the exact solution. The exact analytical solution of the test problem, which is not given explicitly here, corresponds to the Orowan bowing of the continuously distributed dislocations that take up circular-arc configurations, the radii of which decrease with the increasing load. We note in passing that the Orowan bowing of dislocations with line tension is responsible for the size effect in the present model. Unlike as in a local theory, the distribution and amount of plastic slip in the present model depends on the width $H$ of the plastic channel, cf. Sedláček and Werner [25]. The results are shown in Fig. 10. [Insert Fig. 10] In accord with the above analysis, the numerical solution is indistinguishable from the exact one for slightly bowed-out dislocations where eq. (66) is diffusion dominated. At the load of $0.9 \tau_{\text {Or }}$, the numerically computed plastic slip exhibits slight spurious oscillations. These become quite pronounced at $1 \tau_{\text {Or }}$, especially near the interface where the governing equation (66) becomes strongly convection dominated. We note the boundary layer of the steeply changing orientation in the last loading step that has not been properly resolved. Already the earlier applications of the present model have shown that pronounced boundary layers characterized by steep gradients or even steps in plastic slip occur in the vicinity of impenetrable interfaces [22, 23, 25]. In accord with the above analysis, we observed upcoming spurious oscillations that we have suppressed by using extremely fine discretization of the space variable as well as extremely small time steps, which is computationally very expensive. A more efficient possibility to deal with the unwanted oscillations is to resort to the dislocation-Lagrangian description. This will be done in the next Section.

Before turning to the dislocation-Lagrangian description, let us mention a related inconvenient consequence of considering the present theory in the one-dimensional Eulerian formulation. At an impenetrable interface between an elastic and a plastically deforming materials, a surface dislocation density can be deposited, causing a step-like change in the plastic slip from zero in the elastic material to a finite value in the plastic region [47]. The surface dislocation density was discussed also in the framework of the present model $[22,23,25]$. It is convection-caused and presents a limit case of the boundary layer discussed above. In this case, the orientation at the interface is $\vartheta(0, t)=\pi / 2$, Fig. 9. From 
eq. (65) we see that then, the current density diverges, $\rho\left(x_{1}, t\right) \rightarrow \infty$ for $x_{1} \rightarrow 0$. Referring to the continuum description in the sense of eq. (5), we point out that in the present case even the product $b \rho\left(x_{1}, t\right) \rightarrow \infty$ for $x_{1} \rightarrow 0$. In view of the vanishing velocity at the impenetrable interface, eq. (62), the plastic slip rate there cannot be directly determined, for the product $b \rho(0, t) V(0, t)$ in the Orowan equation $(22)$ is undetermined. In fact, at the interface, this product must be understood in the sense of a limit,

$$
b \rho(0, t) V(0, t)=\lim _{x_{1} \rightarrow 0} b \rho\left(x_{1}, t\right) V\left(x_{1}, t\right),
$$

cf. [23, 25]. As a consequence, despite of the vanishing dislocation velocity at impenetrable interfaces, there can be a non-zero plastic slip rate. The computation of the plastic slip rate (69) is one of the difficulties that are encountered when implementing the one-dimensional Eulerian evolution equations in a numerical method [22, 23, 25]. Simulation of the (physically reasonable) development of the surface dislocation density and the corresponding step in plastic slip is more convenient in the dislocation-Lagrangian approach.

\subsection{Dislocation-Lagrangian evolution equations in 1D}

Dislocation-Lagrangian form of eq. (66) results from eq. (57) as

$$
\frac{\mathrm{d} \vartheta}{\mathrm{d} t}=\frac{\partial V}{\partial x_{1}} \cos \vartheta
$$

whereas the density is still computed from eq. (65). The plastic slip rate is computed from eq. (58) that can be further simplified as follows. The plastic slip equals the Burgers vector multiplied by the area swept by the dislocations from the reference configuration represented by the homogeneous density $\bar{\rho}$,

$$
\gamma^{\mathrm{p}}=b \bar{\rho} \varphi_{2}
$$

where $\varphi_{2}\left(x_{1}, t\right)$ is the current displacement of a dislocation segment positioned at $x_{1}$, Fig. 11. [Insert Fig. 11] The convective derivative in eq. (58) thus becomes

$$
\operatorname{grad} \gamma^{\mathrm{p}} \cdot \boldsymbol{V}=-b \bar{\rho} V \sin \vartheta \frac{\partial \varphi_{2}}{\partial x_{1}}
$$

Recognizing that $\partial \varphi_{2} / \partial x_{1}=\tan \vartheta$ and utilizing eq. (65), we obtain from eq. (58) the plastic slip rate in terms of the current, $\rho\left(x_{1}, t\right)$, or reference, $\bar{\rho}$, dislocation density as

$$
\frac{\mathrm{d} \gamma^{\mathrm{p}}}{\mathrm{d} t}=b \rho V(\cos \vartheta)^{2}=b \bar{\rho} V \cos \vartheta
$$

This result can be understood as follows. Looking at Fig. 11 that shows two consecutive positions of a gliding dislocation, we can interpret the result in terms of the rate of change 
in the area swept by the dislocations. In the one-dimensional framework, the change in position $\varphi(X, t)$ of a dislocation segment $X$ that was in time $t$ at $x_{1}$ and which glides in the direction perpendicular to itself results from eq. (52) as

$$
\frac{\mathrm{d} \varphi_{1}}{\mathrm{~d} t}=-V \sin \vartheta, \quad \frac{\mathrm{d} \varphi_{2}(X, t)}{\mathrm{d} t}=V \cos \vartheta
$$

The change in dislocation displacement $\varphi_{2}\left(x_{1}, t\right)$ at the position $x_{1}$ can be formally determined from eq. (53) which yields in this case

$$
\frac{\mathrm{d} \varphi_{2}}{\mathrm{~d} t}=\frac{\partial \varphi_{2}}{\partial t}-\frac{\partial \varphi_{2}}{\partial x_{1}} V \sin \vartheta
$$

With $\partial \varphi_{2} / \partial x_{1}=\tan \vartheta$ we have

$$
\frac{\partial \varphi_{2}}{\partial t}=\frac{V}{\cos \vartheta}
$$

Now we get the dislocation-Lagrangian plastic slip rate as the rate of change in the area swept by the dislocations,

$$
\frac{\mathrm{d} \gamma^{\mathrm{p}}}{\mathrm{d} t}=b \bar{\rho} \frac{\mathrm{d} \varphi_{2}}{\mathrm{~d} t}=b \bar{\rho} V \cos \vartheta,
$$

which reproduces eq. (71). As for the Eulerian plastic slip rate, upon using relation (65), we get analogously

$$
\frac{\partial \gamma^{\mathrm{p}}}{\partial t}=b \bar{\rho} \frac{\partial \varphi_{2}}{\partial t}=b \rho V,
$$

which is the Orowan equation (23). It should be noted that the result (71) is 1D specific, whereas eq. (58) is valid generally.

Let us now analyze the dislocation-Lagrangian eq. (70) from the point of view of the convection-diffusion ratio. Treating it in the same way as the Eulerian eq. (66), we get

$$
\frac{\mathrm{d} \vartheta}{\mathrm{d} t}=\frac{T(\cos \vartheta)^{2}}{B} \frac{\partial^{2} \vartheta}{\partial x_{1}^{2}}+\frac{T \sin \vartheta \cos \vartheta}{B}\left(\frac{\partial \vartheta}{\partial x_{1}}\right)^{2}
$$

The first term on the right-hand side is the same diffusive one as in the corresponding Eulerian eq. (68), the second one is a non-linear term of the convection type. The ratio

$$
\frac{\Delta x \sin \vartheta}{\cos \vartheta}
$$

of the coefficients of the convective (multiplied with $\Delta x$ ) and diffusive terms ${ }^{4}$ goes to infinity for $\vartheta \rightarrow \pi / 2$ and constant $\Delta x$. However, because of the dislocation-Lagrangian description, $\Delta x \rightarrow 0$ in the boundary layer in this case, cf. Fig. 10. As a consequence, the convective term does not dominate, not even for strongly bowed-out dislocations near the impenetrable interface.

\footnotetext{
${ }^{4}$ Because of the nonlinearity in eq. (73) it is not exactly the dimensionless Péclet number itself.
} 
The suitability of the dislocation-Lagrangian description for the present model is certified by performing the computation of the test problem that was posed in Sec. 5.1. Equations (70), (71), and (72) $)_{1}$ have been solved numerically using central differentiation on 13 selfadjusting nodes and an implicit time integration with step-size control up to equilibrium in the individual loading steps. The results plotted in Fig. 10 show an agreement with the exact solution up to the load of $0.9 \tau_{\text {Or }}$ and a slight departure from the analytically calculated plastic slip for the maximum load $1 \tau_{\text {Or }}$, but no oscillations at all. The originally equidistantly positioned nodes have moved along the characteristics during the computation, cf. eq. (72). As a consequence of this node redistribution, even the boundary layer of steeply changing orientation near the interface $(x=0)$ has been sufficiently resolved. The dislocation-Lagrangian node redistribution corresponds to the physical requirement that dislocation segments glide in the direction normal to themselves, $\boldsymbol{V}=V \boldsymbol{\nu}$, which results in the convective transport of plastic slip to the interface. At a further straining, this would result in the surface dislocation density and a step in plastic slip at the impenetrable interface that was discussed in relation with eq. (69). The dislocation-Lagrangian description thus reflects the physical features of the present model of evolving dislocation fields and its implementation is numerically stable.

\section{Conclusions}

In Sec. 2, we have analyzed the deficiencies of the continuum theory of dislocations when applied to set up a constitutive law for crystal plasticity. These deficiencies are due to the process of averaging in which individual dislocations are smeared out so that the shortrange elastic fields of the original dislocations disappear and their curvature cannot be reconstructed any more. Based on our earlier proposals [22, 23, 25], we have suggested in Sec. 3 that this problem can be circumvented to some extent by introducing the concept of single-valued dislocation fields together with the dislocation line tension. In this approach, the dislocation configuration is supposed to consists of a superposition of dislocation fields of unique orientation. In this way, the dislocation curvature can be reconstructed and the line-tension concept applied, leading to a non-local constitutive law, where the plastic slip rate results from the evolution of the dislocation fields. Equations governing the evolution of the dislocation density and orientation have been derived together with the equation for the resulting plastic slip rate in Sec. 4. In the Eulerian approach that describes the evolution of the fields at fixed positions in space, the latter equation is the standard Orowan relation, $\dot{\gamma}=b \varrho V$. In the dislocation-Lagrangian approach, proposed already by Sedláček et al. [23], moving along with the dislocation segments, one follows the net development of the fields. In the present work, we have shown that in the dislocationLagrangian description, the Orowan relation acquires an additional term, namely the convective derivative of plastic slip, see eq. (58). The equations have been applied to an example of expanding dislocation loops, showing that the equations account correctly for the increasing length of the continuously distributed bowed gliding dislocations. 
The theory has been specialized to one spatial dimension in Sec. 5. In this form, the field variables (the orientation and density of the dislocations as well as the plastic slip) are functions of the slip direction only. This approach enables us to treat two-dimensional plane-strain problems of continuum mechanics, where the slip planes are perpendicular to the plane of deformation and the dislocations bow out in their glide planes, cf. Fig. 8 . The equation governing the evolution of the dislocation orientation has turned out to be a transport equation of diffusion-convection type. As the dislocations bow out, the convection dominates in the vicinity of impenetrable interfaces, leading to pronounced spurious oscillations in the resulting plastic slip. This numerical instability which is known from the mathematical literature has been eliminated by resorting to the dislocation-Lagrangian description resulting in a kind of dislocation-Lagrangian particle-tracking method, cf. Fig. 10.

In the present work, we have focused our attention on the constitutive part of the problem of plastic deformation. The obtained results on the non-local dislocation-based constitutive law of plasticity, especially the numerical stability of the dislocation-Lagrangian approach, enable us to start a work on numerical solution of size-dependent two-dimensional plane-strain problems of continuum mechanics with non-trivial geometry, morphology, and boundary conditions.

\section{Acknowledgements}

The authors are grateful for the financial support by grants DFG We 2351/8-3 (RS, CS, EW) and VZ-MŠMT 6840770003 (JK).

\section{A Solution to equation (45)}

We are looking for the solution $\rho(r, t)$ of the quasilinear partial differential equation (45) in the form

$$
\frac{\partial \rho}{\partial t}+\left(\frac{a r-c}{r}\right) \frac{\partial \rho}{\partial r}+\rho \frac{c}{r^{2}}=0
$$

where we have used eq. (40) and introduced the constants

$$
a=\frac{\bar{\tau} b}{B}, \quad c=\frac{T}{B} .
$$

We use the method of characteristics, also known as the Lagrange method. The characteristic curves in the $r$ - $t$ plane are defined by eq. (46) that becomes

$$
\frac{\mathrm{d} r}{\mathrm{~d} t}=\frac{a r-c}{r} .
$$


Equation describing the characteristic curves results by integration as,

$$
t(r)=\frac{r}{a}+\frac{c}{a^{2}} \ln (|a r-c|)+C .
$$

To determine the integration constant $C$, put $t=0$ in the above equation and choose a position on the $r$ axis. For instance, setting $r=R_{1}$ and $r=R_{2}$, we get the constants $C_{1}$ and $C_{2}$ of the characteristics drawn in the lower part of Fig. 5 . The inverse of the above equation of the characteristics yields

$$
r(t)=\frac{c}{a}\left(1+W\left(\frac{1}{c} \exp \left(\frac{a^{2}(t-C)-c}{c}\right)\right)\right)
$$

where $W(f)$ is the Lambert $W$-function which is the inverse of the function $f(W)=$ $W \exp (W)$.

The general solution to eq. (A1) can be written as

$$
\rho(r, t)=\frac{r}{c-a r} g\left(t-\frac{r}{a}-\frac{c}{a^{2}} \ln (|a r-c|)\right),
$$

where $g$ is a function to be determined so that an initial condition $\rho(r, 0)=\rho_{0}(r)$ be fulfilled. From the general solution (A2) follows the initial condition in the form

$$
\rho_{0}(r)=\frac{r}{c-a r} g\left(-\frac{r}{a}-\frac{c}{a^{2}} \ln (|a r-c|)\right) .
$$

We look for the function $g(\xi)$, where we have introduced a new variable $\xi(r)$ such that

$$
\xi=-\frac{r}{a}-\frac{c}{a^{2}} \ln (|a r-c|),
$$

with the inverse

$$
r(\xi)=\frac{c}{a}\left(1+W\left(\frac{1}{c} \exp \left(-\frac{a^{2} \xi+c}{c}\right)\right)\right) .
$$

From eq. (A3) we get

$$
g(\xi)=\rho_{0}(r(\xi)) \frac{c-a r(\xi)}{r(\xi)} .
$$

To get the general solution (A2), we insert the expression $r(\xi)$ in the above equation, while using for the variable $\xi$ the corresponding expression from eq. (A2),

$$
\xi=\left(t-\frac{r}{a}-\frac{c}{a^{2}} \ln (|a r-c|)\right) .
$$

The general solution results finally as

$$
\rho(r, t)=-\frac{a r}{c-a r} \rho_{0}\left[\frac{c}{a}\left(1+W\left(\frac{a r-c}{c} \exp \left(\frac{a r-a^{2} t-c}{c}\right)\right)\right)\right] \frac{W\left(\frac{a r-c}{c} \exp \left(\frac{a r-a^{2} t-c}{c}\right)\right)}{1+W\left(\frac{a r-c}{c} \exp \left(\frac{a r-a^{2} t-c}{c}\right)\right)} .
$$


Note that the expression in square brackets is the argument of the function $\rho_{0}$. Using this solution with the initial condition (38), we have produced the results that are plotted in the upper part of Fig. 5.

Moreover, arbitrary initial conditions can be considered when using this general solution. As an example, we calculated the solution for a parabolic initial distribution of the concentric loops as well, see Fig. 12. [Insert Fig. 12]

\section{References}

[1] N. A. Fleck, G. M. Muller, M. F. Ashby, and J. W. Hutchinson, Acta Metall. Mater. 42475 (1994).

[2] E. Arzt, Acta Mater. 465611 (1998).

[3] J. S. Stölken and A. G. Evans, Acta Mater. 46:5109 (1998).

[4] J. W. Hutchinson, Int. J. Solids and Structures 37225 (2000).

[5] A. Needleman, Acta Mater. 48105 (2000).

[6] L. P. Kubin and G. Canova, Scripta Metall. Mater. 27957 (1992).

[7] B. Devincre and L. P. Kubin, Mater. Sci. Eng. A 234-236 8 (1997).

[8] E. Van der Giessen and A. Needleman, Modelling Simul. Mater. Sci. Eng. 3689 (1995).

[9] M. C. Fivel and A. El-Azab, Journal de Physique IV Pr9 261 (1999).

[10] C. Lemarchand, B. Devincre, and L. P. Kubin, J. Mech. Physics Solids 491969 (2001).

[11] V. Bulatov, J. Computer-aided materials design 9133 (2002).

[12] E. Kröner, in Inelastic behavior of solids, edited by M. F. Kanninen, W. F. Adler, A. R. Rosenfeld, and R. I. Jaffee (McGraw-Hill, 1969), p. 137.

[13] I. Groma, Phys. Rev. B 565807 (1997).

[14] I. Groma, F. F. Csikor, and M. Zaiser, Acta Mater. 511271 (2003).

[15] S. Yefimov, I. Groma, and E. van der Giessen, J. Mech. Physics Solids 52279 (2002).

[16] E. Kröner, Kontinuumstheorie der Versetzungen und Eigenspannungen (Springer, Berlin, 1958). 
[17] E. Kröner, in Physics of Defects, edited by R. Balian, M. Kléman, and J. P. Poirier (NATO ASI, North Holland 1981).

[18] A. M. Kosevich, Soviet Physics Uspekhi 7837 (1965).

[19] A. M. Kosevich, in Dislocations in Solids, edited by F. R. N. Nabarro. Volume 1, The Elastic Theory (North-Holland, Amsterdam, 1979), p. 33.

[20] T. Mura, Int. J. Eng. Science 1371 (1963).

[21] T. Mura, Philos. Magazine 8843 (1963).

[22] R. Sedláček and S. Forest, phys. stat. sol. (b) 221583 (2000).

[23] R. Sedláček, J. Kratochvíl, and E. Werner, Philos. Magazine 833735 (2003).

[24] J. Kratochvíl and R. Sedláček, Phys. Rev. B 67094105 (2003).

[25] R. Sedláček and E. Werner, Phys. Rev. B 69134114 (2004).

[26] V. John and P. Knobloch, Comp. Methods Appl. Mech. Eng., submitted (Preprint Nr. 156, FR 6.1 - Mathematik, Universitt des Saarlandes, Saarbrcken, 2005).

[27] A. Smolianski, O. Shipilova and H. Haario, Research report 98, Laboratory of Applied Mathematics, Lappeenranta University of Technology, 2005.

[28] A. Arsenlis, D.M. Parks, R. Becker and V.V. Bulatov, J. Mech. Phys. Solids 521213 (2004).

[29] A. Roy and A. Acharya, J. Mech. Phys. Solids 53143 (2005).

[30] Y. Xiang, L. Cheng, D.J. Srolovitz and E. Weinan, Acta Mater. 515499 (2003).

[31] E. Kröner, Int. J. Solids Structures 381115 (2001).

[32] R. W. Ogden, Non-linear Elastic Deformation (John Wiley, Horwood, 1984).

[33] J.P. Hirth and J. Lothe, Theory of Dislocations (John Wiley, 1982).

[34] J. Kratochvíl and M. Saxlová, Scripta Metall. Mater. 26113 (1992).

[35] R. Sedláček, Scripta Metall. Mater. 33283 (1995).

[36] A. El-Azab, Phys. Rev. B 6111956 (2000).

[37] R. Sedláček, Mater. Sci. Eng. A 393387 (2005)

[38] C. Schwarz and R. Sedláček and E. Werner, Model. Simul. Mater. Sci. Eng. (accepted)

[39] G. deWit and J.S. Koehler, Phys. Rev. 1161113 (1959). 
[40] J. Kratochvíl and R. Sedláček, Proc. 2nd Int. Conf. Multiscale Materials Modeling, ed. N.M. Ghoniem, p. 221, UCLA (2004).

[41] L.P. Kubin, C. Fressengeas and G. Anantakrishna, in Dislocations in Solids, vol.11, eds. F.R.N. Nabarro and M.S. Duesbery, (Elsevier Science, Amsredam, 2002), p. 101.

[42] R.J. Asaro, Acta Metall. 27445 (1979).

[43] R.J. Asaro, J. Appl. Mechanics 50921 (1983).

[44] M. Zaiser and A. El-Azab, Mat. Res. Soc. Symp. Proc. 779 W5.7.1 (2003).

[45] W. D. Nix, Metall. Transactions A 20A 2217 (1989).

[46] C. Schwarz, R. Sedláček, and E. Werner, Mater. Sci. Eng. A 400-401 443 (2005).

[47] H. Mughrabi, Acta Metall. 311367 (1983). 


\section{Figure captions}

Figure 1: Coupling of the continuum theory of evolving dislocation fields with the continuum-mechanics framework. The input to the constitutive law is the resolved shear stress $\tau$ that drives the dislocation motion. The theory yields the plastic strain rate $\partial \boldsymbol{\beta}^{\mathrm{p}} / \partial t$ resulting from this motion.

Figure 2: Dislocation loops distributed homogeneously in a plastic channel (left). The corresponding geometrically necessary density (right).

Figure 3: Sketch illustrating the inflow of dislocations in a test area $A$. 
Figure 4: Initial configuration of the continuously distributed expanding loops. The polar coordinates used and the corresponding base vectors are shown.

Figure 5: Exact analytical solution to eq. (45) with initial condition (38). In the upper part, snapshots of the evolving dislocation density are plotted. The lower part shows the characteristics in the $r$ - $t$ plane belonging to the boundaries $R_{1}$ and $R_{2}$ of the initial interval of non-zero dislocation density. The characteristics result as a solution to eq. (46), cf. Appendix A. The time levels corresponding to the individual snapshots are indicated, showing how the characteristics determine the boundaries of the evolving interval of the non-zero dislocation density.

Figure 6: Numerically computed dislocation density and plastic slip caused by the expanding loops. The left part of the figure corresponds to the correctly expanding loops of increasing length: the plastic slip in the points $r$ passed by all the loops is constant. The right part represents disintegrating loops of constant total length: the plastic slip in the points $r$ passed by all the loops in later times $t$ is smaller. Heavy lines: 10000 nodes spaced regularly along the $r$ axis. Faint dashed lines: 1000 nodes.

Figure 7: Snapshots of the evolving dislocation density and plastic slip. As in Fig. 6, the left part of the figure corresponds to the correctly expanding loops of increasing length. The right part represents disintegrating loops of constant total length. Heavy lines: dislocation-Lagrangian solution on 100 self-adjusting nodes. Faint dashed lines: Eulerian solution on 10000 nodes from Fig. 6. The Lagrange solution is defined only at the current position of the nodes. It coincides with the exact analytical solution plotted in Fig. 5 .

Figure 8: A threading dislocation in a free-surface thin film on a substrate (after Nix [45]). Assuming a homogeneous density of continuously distributed dislocations in the sample, a coupled dislocation-mechanics plane-strain problem can be set up. Depending on the mechanical boundary conditions, such a problem can be possibly further reduced to one spatial dimension in the $z$ direction, cf. $[25,46]$. 
Figure 9: A typical bowed-out configuration of a representative dislocation in a glide plane, between an impenetrable interface $(x=0)$ and free surface $(x=H)$. The reference configuration, cf. eq. (65), is represented by the straight dashed line.

Figure 10: The test problem of a one-dimensional field of dislocations bowing in a homogeneous shear stress between an impenetrable interface $(x=0)$ and a free surface $(x=1 \mu \mathrm{m})$, computed in the Eulerian (left) and dislocation-Lagrangian (right) approaches. Plotted is the resulting plastic slip $\gamma^{\mathrm{P}}(x)$ and dislocation orientation $\vartheta(x)$. The nodes used for the computation are indicated by circles. The fine dotted lines represent the exact solution.

Figure 11: Eulerian (left) and dislocation-Lagrangian (right) increment in position $\varphi_{2}\left(x_{1}, t\right)$ of (and the area swept by) a dislocation segment, leading to the difference in the Eulerian and dislocation-Lagrangian plastic slip rate.

Figure 12: Exact analytical solution to eq. (45) with a parabolic initial condition. A numerical calculation based on the dislocation-Lagrangian method has yielded identical results. 


\section{Page 37 of 48 Philosophical Magazine \& Philosophical Magazine Letters}

\section{initial dislocation distribution}

boundary conditions (dislocations)

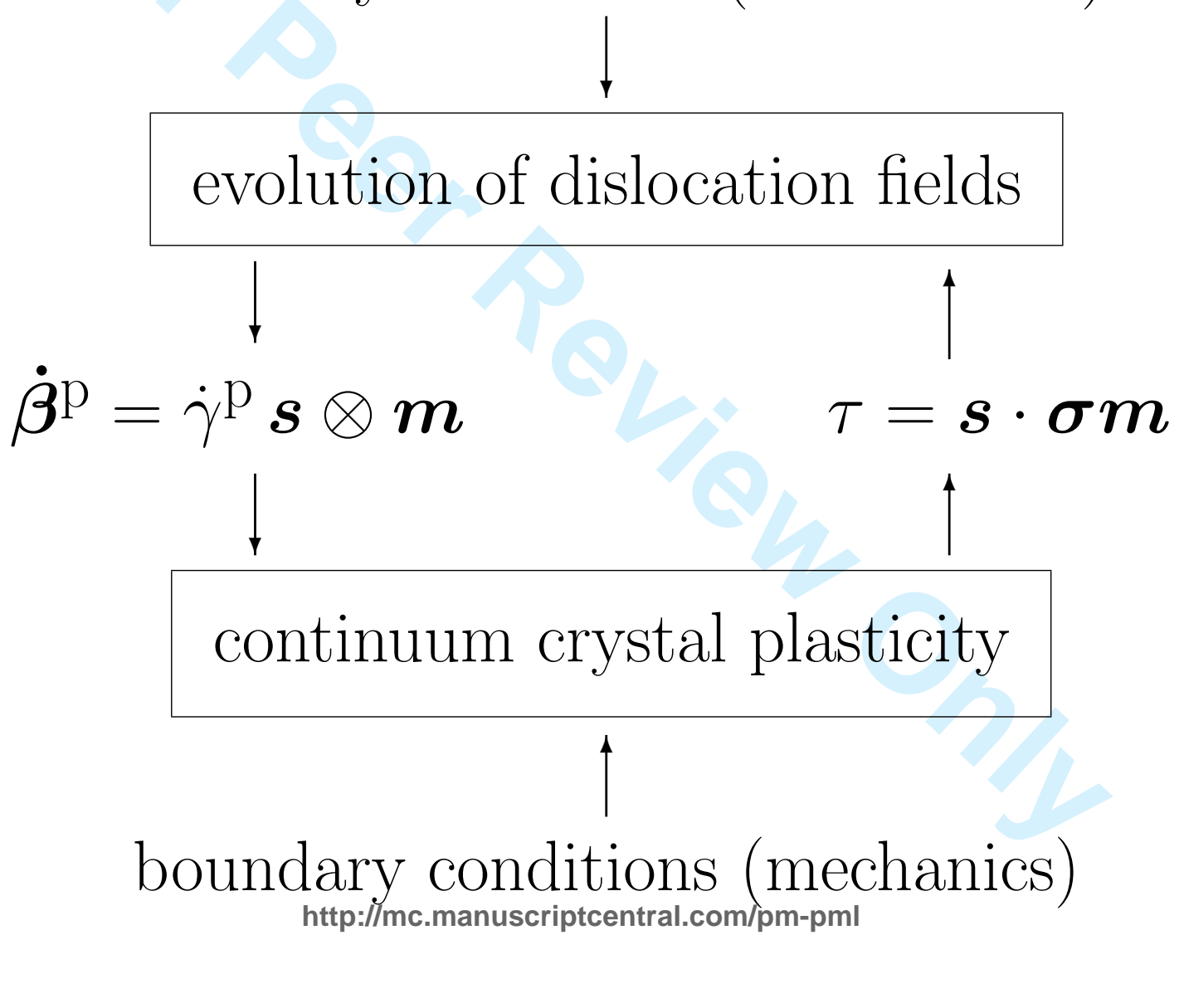




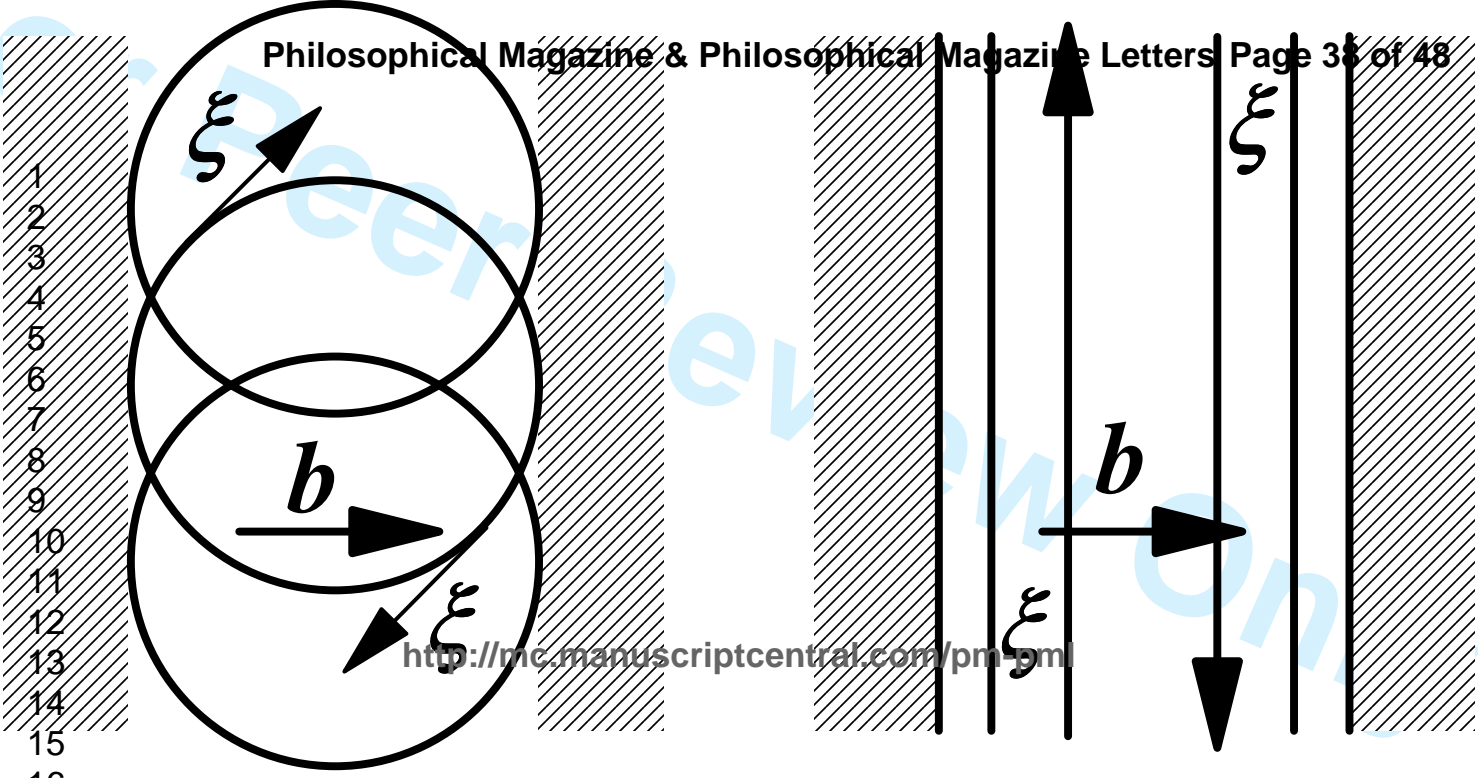




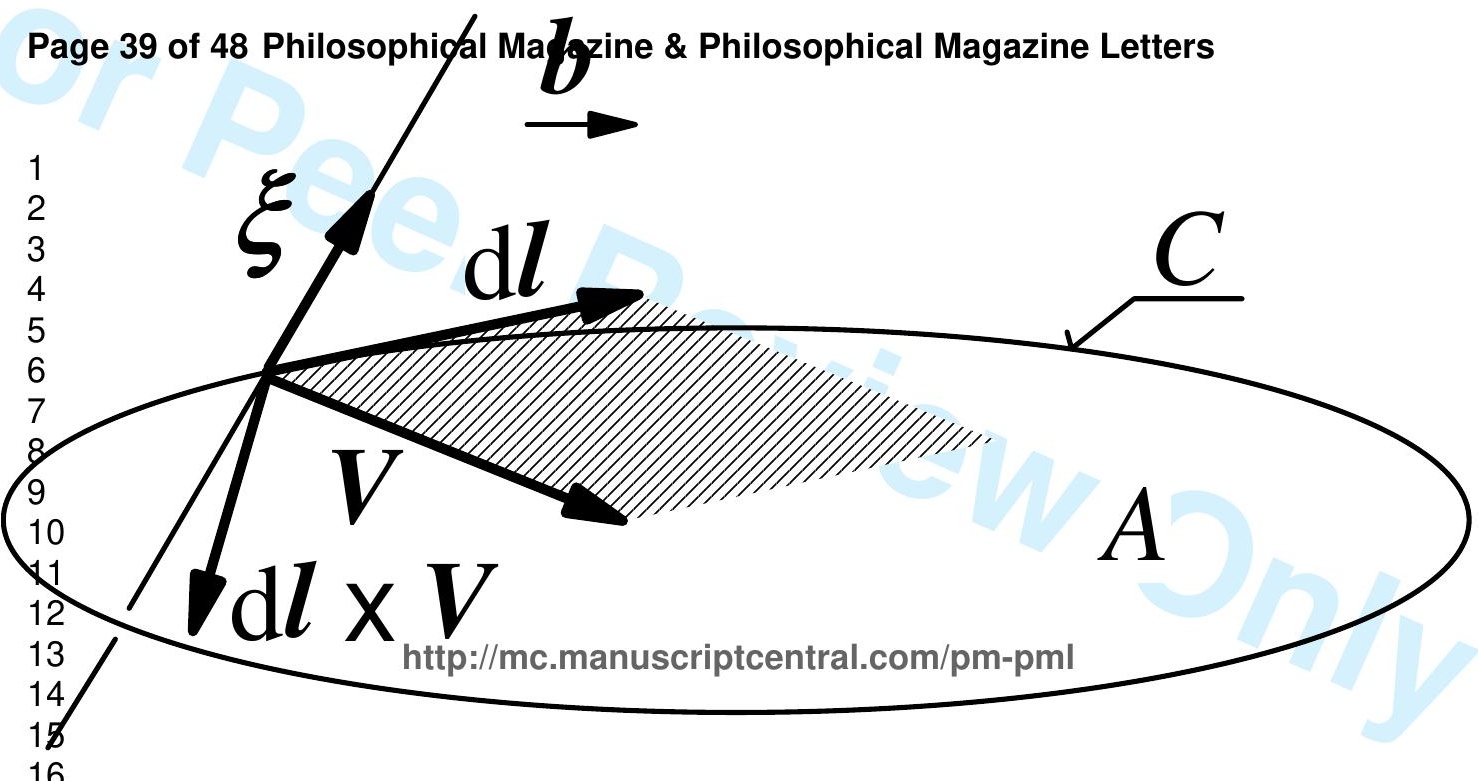





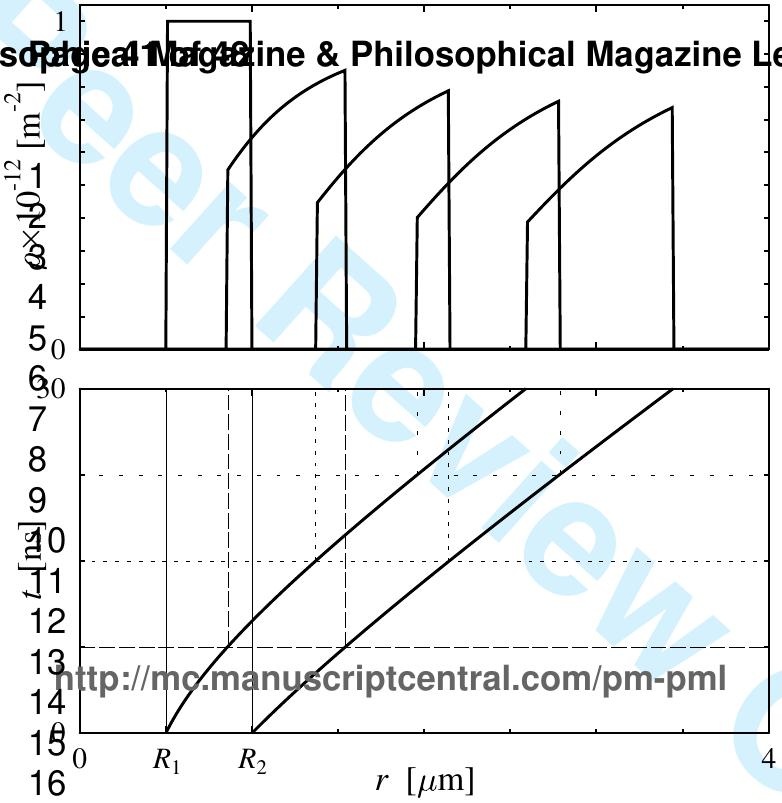




\section{Expanding loops
Disintegrating loops \\ Expanding loops
Disintegrating loops}

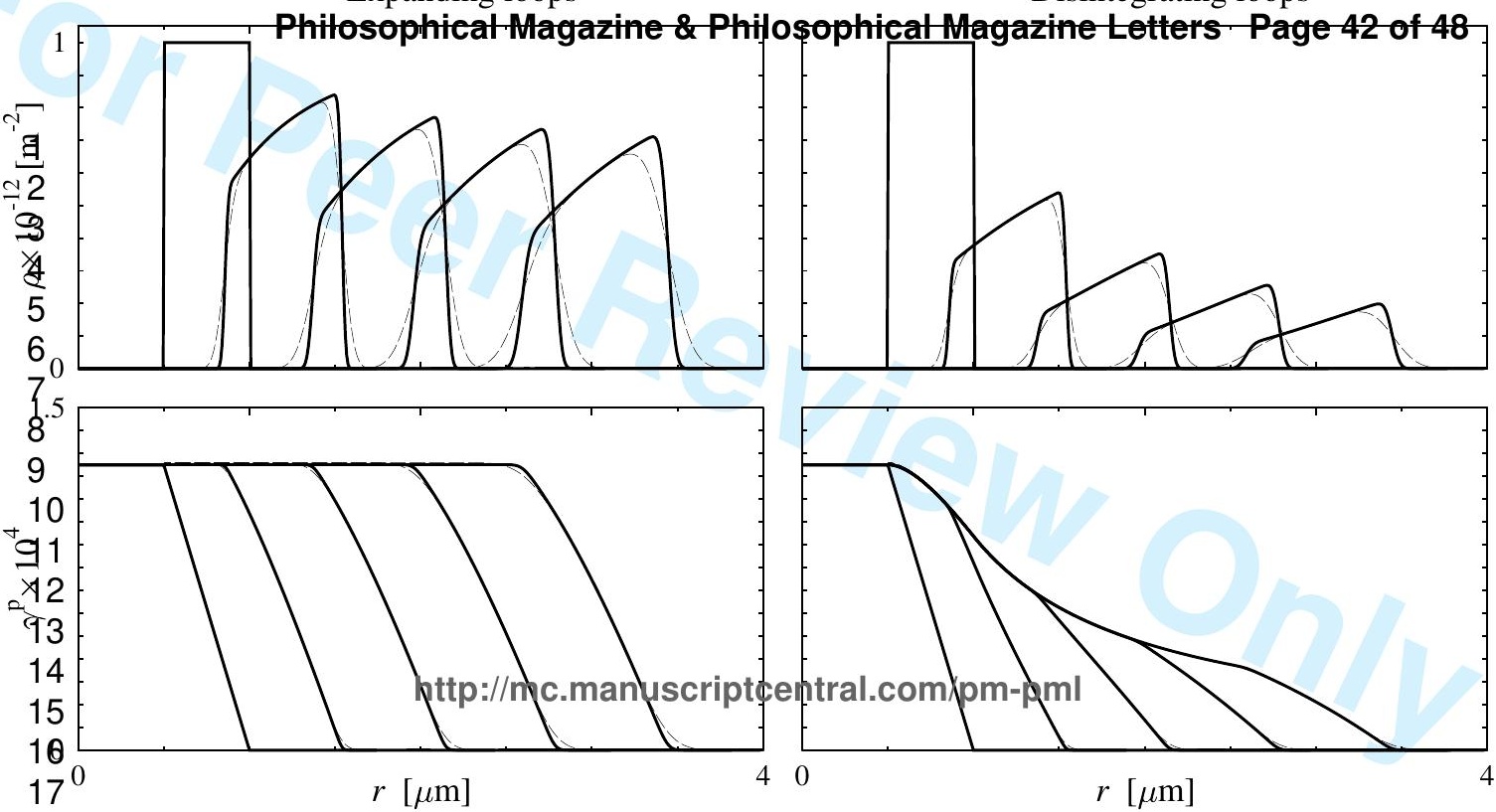


Expanding loops

Disintegrating loops

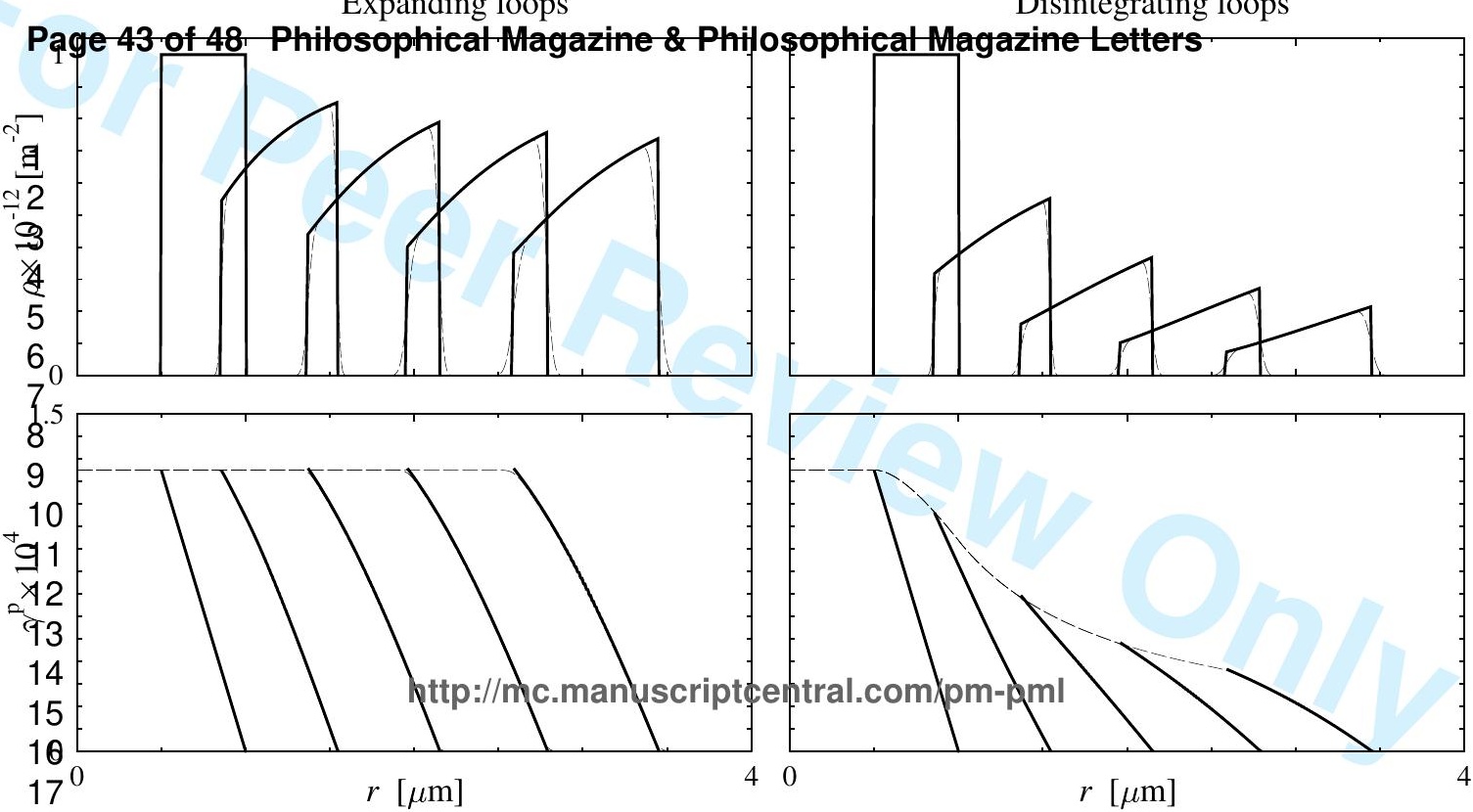




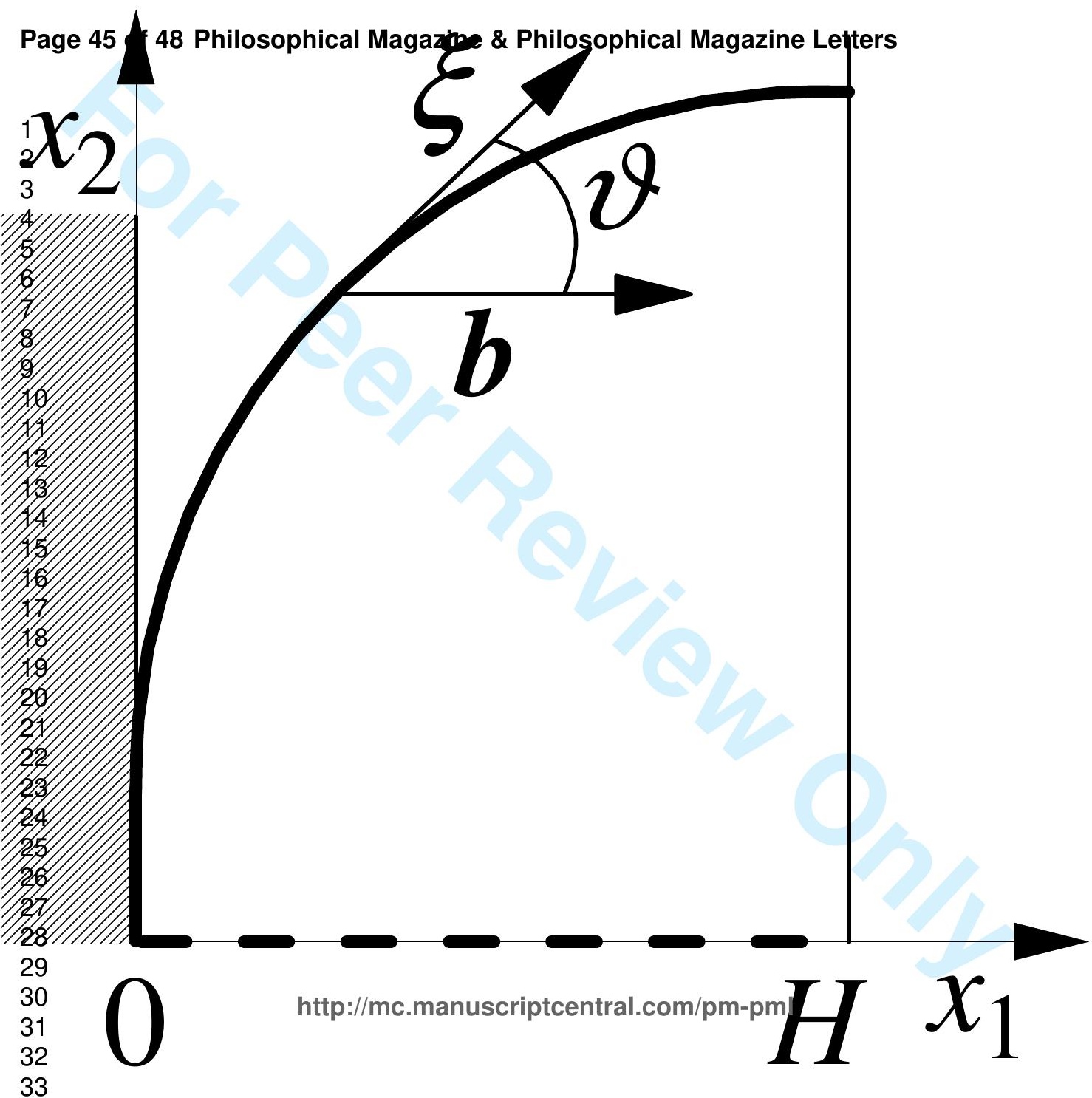


Eulerian approach

Lagrangian approach

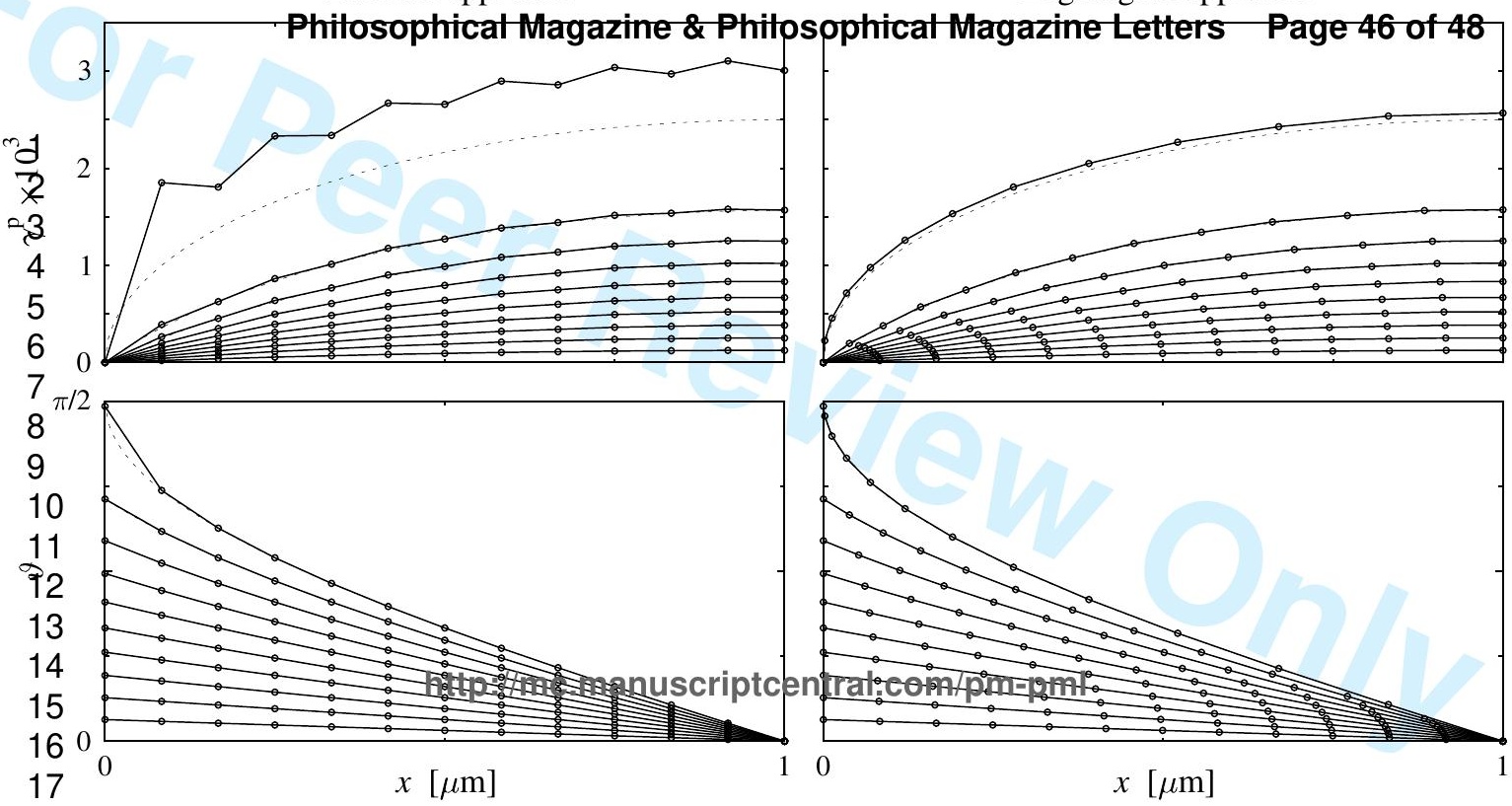




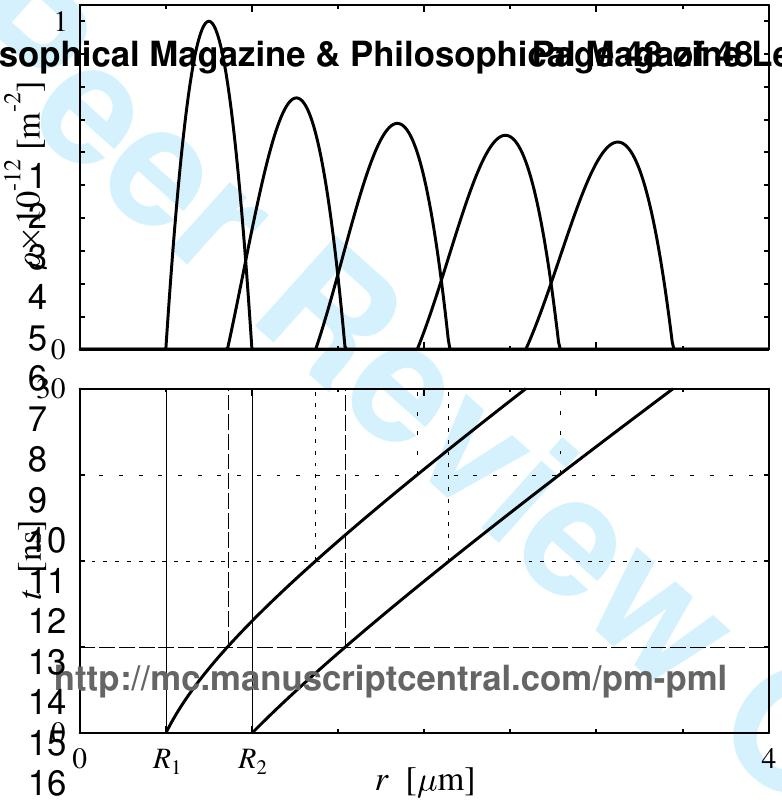

